\title{
Multifunctional Ginger Nanofiber Hydrogels with Tunable Absorption: The Potential for Advanced Wound Dressing Applications
}

\author{
Paula Squinca, Linn Berglund,* Kristina Hanna, Jonathan Rakar, Johan Junker, Hazem Khalaf, \\ Cristiane S. Farinas, and Kristiina Oksman
}

Cite This: Biomacromolecules 2021, 22, 3202-3215

Read Online

ACCESS | Llll Metrics \& More | 回 Article Recommendations | st Supporting Information

ABSTRACT: In this study, ginger residue from juice production was evaluated as a raw material resource for preparation of nanofiber hydrogels with multifunctional properties for advanced wound dressing applications. Alkali treatment was applied to adjust the chemical composition of ginger fibers followed by TEMPO (2,2,6,6-tetramethylpiperidine-1-oxyl radical)-mediated oxidation prior to nanofiber isolation. The effect of alkali treatment on hydrogel properties assembled through vacuum filtration without addition of any chemical cross-linker was evaluated. An outstanding absorption ability of $6200 \%$ combined with excellent mechanical properties, tensile strength of $2.1 \pm 0.2 \mathrm{MPa}$, elastic modulus of $15.3 \pm 0.3 \mathrm{MPa}$, and elongation at break of $25.1 \%$, was

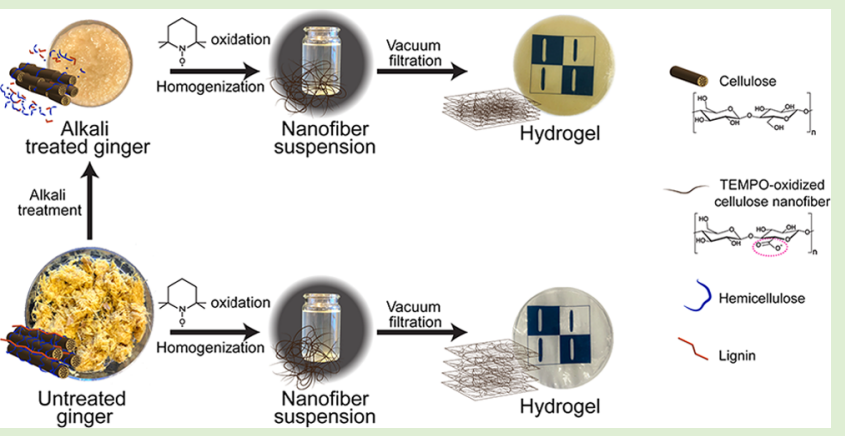
achieved without alkali treatment. Furthermore, the absorption capacity was tunable by applying alkali treatment at different concentrations and by adjusting the hydrogel grammage. Cytocompatibility evaluation of the hydrogels showed no significant effect on human fibroblast proliferation in vitro. Ginger essential oil was used to functionalize the hydrogels by providing antimicrobial activity, furthering their potential as a multifunctional wound dressing.

\section{INTRODUCTION}

Advanced wound dressings not only passively cover wounds but also provide functions that promote healing or improve wound care. An ideal wound dressing should provide a moist environment, absorb exudates, and protect against bacterial infection, while also being biocompatible and mechanically stable. ${ }^{1,2}$ It is generally accepted that a moist environment facilitates the healing of acute and chronic wounds ${ }^{3-5}$ and also facilitates epidermal cell migration, increasing the rate of reepithelialization and angiogenesis. ${ }^{6,7}$

Hydrogels have been considered a suitable material for moist wound dressings since they absorb and retain water, which provides a moist environment for the wound. Hydrogels can have properties such as nonadhesion, biocompatibility, and transparency. These properties can be leveraged to develop advanced wound dressings with superior and novel functions, such as enabling continuous monitoring of the wound without removal of the dressing. ${ }^{7-10}$ The low absorbent capability of some hydrogels geared toward wound dressings has encouraged the development of new "superabsorbent" products that exhibit high degrees of swelling, often well over 10 times their dry mass. ${ }^{11}$ This property may be useful for high rates of wound exudate absorption, which may be important for wound dressing applications. ${ }^{12}$
The degree of hydration can change the mechanical properties of hydrogels, often resulting in worse mechanical stability in a swollen state. This can limit their practical application as wound dressings. ${ }^{13}$

2,2,6,6-Tetramethylpiperidine-1-oxyl (TEMPO)-oxidized cellulose nanofibers present attractive characteristics, such as small and uniform widths (around $3 \mathrm{~nm}$ ) and high aspect ratio (higher than 150), that distinguish them from nonoxidized nanofibers that can provide transparent and strong network formation with increased mechanical performance. ${ }^{14}$ In recent years, nanofiber-based hydrogels have been exploited for wound dressing applications. ${ }^{15-20}$ However, to achieve the desired multifunctional properties, such as swelling combined with mechanical stability, the preparation procedures of these hydrogels are often complex involving cellulose nanofibers in combination with several different components such as

Received: February 16, 2021

Revised: July 1, 2021

Published: July 13, 2021 
alginate, $^{16}$ chitosan, ${ }^{17,19}$ and cross-linking agents ${ }^{16,19}$ for the stability.

The use of silver nanoparticles in wound dressings is a wellknown approach for minimizing microbial contamination of wounds and achieving antimicrobial materials. ${ }^{15}$

However, silver nanoparticles can be difficult to control and their use is known to have side effects on patient health. ${ }^{21}$ Biobased materials with novel, nature-derived antimicrobial properties have recently been explored as an important advanced functionalization of wound dressings. ${ }^{22}$ This approach can reduce the microbial challenge to wounds, thereby minimizing the risks associated with wound infections, all the while avoiding the further use of classical antibiotics, the use of which exasperates the threat of antimicrobial resistance. $^{22}$

Zingiber officinale Roscoe, commonly known as ginger, is extensively used as a spice, but it has also been used as traditional medicine due to its purported antioxidant, antiviral, antidiabetic, anti-inflammatory, anticancer, as well as antibacterial properties. ${ }^{23-27}$ Ginger is rich in constituents such as cellulose, starch, and hemicellulose but also includes several bioactive families of compounds, such as gingerols, zingiberene, and shogaols. ${ }^{28}$ Ginger essential oil (GEO), mainly composed of geranial, $\alpha$-zingiberene, (E,E)- $\alpha$-farnesene, neral, and ar-curcumene, has also shown significant antimicrobial, antifungal, and antioxidant activities. ${ }^{29,30}$ Although the pharmacological properties have been supported by in vivo and in vitro experiments, ${ }^{31}$ only a few studies have focused on using its nanofibers for the preparation of bionanocomposites, $^{32-34}$ films, ${ }^{35-37}$ and aerogels. ${ }^{38,39}$ The potential use of ginger and its natural components for nanofiber extraction and their utilization for the preparation of completely ginger-based hydrogels have, so far, not been studied for wound dressing applications.

In this study, we investigated the potential use of ginger for nanofiber extraction and subsequent assembly into hydrogels aimed at wound dressing applications. The hydrogels were prepared by simple vacuum-assisted filtration using only ginger nanofibers without any cross-linker to maintain low energy requirements, minimize the components needed for sustainable production, and avoid the risk of introducing toxic side effects. Alkali treatment was applied on ginger fibers before TEMPO oxidation to modify their chemical composition, altering the liquid absorption capacity of the hydrogels, which is evaluated in water, bovine serum albumin (BSA), and phosphate-buffered saline (PBS) solutions. The functionalization of ginger-based hydrogels with ginger essential oil seems like an advantageous strategy because it has the potential to leverage the antimicrobial properties of the plant to enhance the antimicrobial properties of the wound dressing while being sourced from the same raw materials. The structural morphology, mechanical properties in wet conditions, cytocompatibility, and antimicrobial properties of two versions of ginger nanofiber (GNF) hydrogels were quantified, supporting an initial evaluation of these materials for use in advanced wound dressing products.

\section{EXPERIMENTAL SECTION}

Materials. Ginger roots were purchased from a local market, and the fibers were obtained after juicing and used as the feedstock in this study. High-purity sodium chlorite $\left(\mathrm{NaClO}_{2}\right)$, with a sodium chlorite content of $77.5-82.5 \% \mathrm{w} / \mathrm{w}$, was purchased from VWR International $\mathrm{AB}$ (Stockholm, Sweden). Ginger essential oil, sodium hypochlorite
( $\mathrm{NaClO}, 6-14 \%$ active chlorine), 2,2,6,6-tetramethylpiperidin-1-oxyl (TEMPO, 98\%), bovine serum albumin (BSA) lyophilized, and phosphate-buffered saline (PBS) were purchased from Sigma-Aldrich, Sweden AB (Stockholm, Sweden). Glacial acetic acid $\left(\mathrm{CH}_{3} \mathrm{COOH}\right.$, $100 \%$ ) was purchased from Merck KGaA (Darmstadt, Germany). BSA was used in a concentration of $50 \mathrm{~g} \mathrm{~L}^{-1}$ in distilled water without further treatment.

Alkali Treatment. Alkali treatments were performed using 4 and 2 wt $\% \mathrm{NaOH}$ solutions to alter the chemical composition of the ginger fibers. Treatments were performed at a liquor/dry matter ratio of $80: 1$ and $80^{\circ} \mathrm{C}$ for $2 \mathrm{~h}$ under magnetic stirring. Sequentially, alkalitreated ginger fibers were washed with distilled water until a neutral $\mathrm{pH}$ was reached.

Bleaching Procedure. Ginger fibers, with and without alkali treatment, were bleached with $\mathrm{NaClO}_{2}(2.5 \mathrm{wt} \%)$ in an acetic buffer ( $\mathrm{pH} 4.5$ ) at $80^{\circ} \mathrm{C}$ for $2 \mathrm{~h}$. After bleaching, the materials were washed with distilled water until a neutral $\mathrm{pH}$ was reached. Sodium chlorite is the primary oxidant in the TEMPO $/ \mathrm{NaClO} / \mathrm{NaClO}_{2}$ system, and bleaching treatments were performed to obtain samples that could be used for estimating the chemical composition of the ginger nanofibers, both with and without alkali treatment.

Chemical Composition. The chemical composition of the raw material, after bleaching of ginger fibers with and without alkali treatment, was determined in accordance with the standard testing recommendations of the Technical Association of Pulp and Paper Industry (TAPPI). The extractive content was determined by Soxhlet extraction with an acetone-alcohol ratio of $2: 1$ for $5 \mathrm{~h}$ following the methodology of T $204 \mathrm{~cm}-97$. $^{40}$ Delignification of all materials was performed according to established protocols ${ }^{41}$ in which three additions of $\mathrm{NaClO}_{2}\left(1 \mathrm{~g} \mathrm{~g}_{\text {dry matter }}{ }^{-1}\right)$ and acetic acid $(0.2 \mathrm{~mL}$ $\left.\mathrm{g}_{\text {dry matter }}{ }^{-1}\right)$ were performed in intervals of $1 \mathrm{~h}$. The reactions were carried out at a liquor/dry matter ratio of $40: 1$ and $70{ }^{\circ} \mathrm{C}$. After delignification, the materials were washed with distilled water until a neutral $\mathrm{pH}$ was reached. Holocellulose and $\alpha$-cellulose contents were determined according to the TAPPI standard T $203 \mathrm{~cm}-99 .{ }^{42}$ The hemicellulose content was calculated as the subtraction of $\alpha$-cellulose from the holocellulose percentage. Klason lignin was determined in a sulfuric acid solution (72 wt \%) following the TAPPI standard T 222 om-02. ${ }^{43}$ The presented component values are based on 10 measurements for each sample, and tests were performed in triplicate.

Yield Determination. The treatment yields (alkali treatment, bleaching, and TEMPO oxidation) were calculated according to the following equation

$$
\text { yield }(\%)=W_{\mathrm{f}} / W_{\mathrm{i}} \times 100
$$

where $W_{\mathrm{f}}$ indicates the dry weight of the sample after the alkali treatment, bleaching, and TEMPO oxidation and $W_{\mathrm{i}}$ indicates the initial dry weight of the ginger fibers.

Optical Microscopy (OM). Characterizations of the ginger fibers before and after the alkali treatment and TEMPO oxidation were performed using an optical microscope, Nikon Eclipse LV100N POL (Kanagawa, Japan), and the imaging software NIS-Elements D 4.30.

Ginger Nanofiber (GNF) Production. Ginger nanofibers were obtained from the TEMPO/ $\mathrm{NaClO} / \mathrm{NaClO}_{2}$ system following a method described by Saito et al. with modifications and using either ginger fibers before or after alkali treatment. ${ }^{44}$ First, $\mathrm{NaClO}_{2}(5.0 \mathrm{~g}$ $\left.\mathrm{g}_{\text {dry matter }}{ }^{-1}\right)$ and TEMPO $\left(17.5 \mathrm{mg} \mathrm{g}_{\text {dry matter }}{ }^{-1}\right)$ were dissolved in the ginger fiber suspension at a liquor/dry matter ratio of 100:1 in the presence of a sodium phosphate buffer $(0.05 \mathrm{M}, \mathrm{pH} 6.8)$. The flask was sealed and submerged in an oil bath, after which $\mathrm{NaClO}(1 \mathrm{~mL}$ $\mathrm{g}_{\text {dry matter }}{ }^{-1}$ ) was added and kept at a temperature of $60^{\circ} \mathrm{C}$ for $72 \mathrm{~h}$. After cooling the suspension to room temperature, the material was washed with distilled water until reaching a neutral $\mathrm{pH}$. After the TEMPO oxidation, the GNF suspension was diluted to $0.2 \mathrm{wt} \%$, homogenized with a high shear fluid homogenizer APV 2000 (SPXFlow Inc, Delavan) at 1000 bar, and collected after 1 pass. Different GNF suspensions were prepared from ginger without alkali treatment after 2 and $4 \mathrm{wt} \%$ alkali treatment, and they are denoted as T-GNF (TEMPO-treated GNF), AT 2\%-GNF, and AT 4\%-GNF (alkali and TEMPO-treated GNF with 2 or $4 \% \mathrm{NaOH}$ ), respectively. 
The surface charge of the ginger nanofibers was measured using a Zetasizer Nano Z (Malvern Pananalytical Ltd, Malvern, U.K.) system at room temperature $\left(25^{\circ} \mathrm{C}\right)$ in triplicate. Prior to the measurements, the samples were diluted to $0.05 \%(\mathrm{w} / \mathrm{v})$ with distilled water.

Preparation of Ginger Nanofiber-Based Hydrogels. Hydrogels with different grammages $\left(40,80\right.$, and $\left.120 \mathrm{~g} \mathrm{~m}^{-2}\right)$ were prepared by vacuum-assisted filtration of ginger cellulose nanofibers on a filter paper (Whatman grade 54, $90 \mathrm{~mm}$ diameter) and metal wire sieve. First, GNF suspensions were diluted to $0.1 \mathrm{wt} \%$ in water and dispersed under magnetic stirring for $10 \mathrm{~min}$. The suspensions were then degassed and poured into a Büchner funnel with a filter paper and metal wire sieve. The suspension was filtered at room temperature $\left(\sim 20{ }^{\circ} \mathrm{C}\right)$ for $8-24 \mathrm{~h}$ depending on the grammage of the hydrogel. After the GNF network was formed, the hydrogels were put into water and peeled from the filter paper. Subsequently, the hydrogels were dried at room temperature $\left(\sim 20{ }^{\circ} \mathrm{C}\right)$, and their swelling degree was adjusted according to the characterization condition tests. A reference hydrogel was prepared from woodbased nanofibers, prepared using TEMPO oxidation and the homogenization treatment described for ginger. The reference hydrogel was prepared at $40 \mathrm{~g} \mathrm{~m}^{-2}$ following an equivalent procedure and used for comparison of the swelling behavior. To enhance the antimicrobial properties of the dressing material, the hydrogels prepared with T-GNF and AT 4\%-GNF were functionalized with ginger essential oil (GEO). Initially, the GNF suspensions were diluted to a concentration of $0.3 \mathrm{wt} \%$ in water and dispersed under magnetic stirring for $10 \mathrm{~min}$. The solution was then placed in a thermostatic oil bath at $40{ }^{\circ} \mathrm{C}$, and GEO (10 wt \%) was added under constant stirring for $20 \mathrm{~min}$. Then, the mixed solution was sonicated using an ultrasonic processor UP400s (Hielscher Ultrasonics GmbH, Teltow, Germany) for 2 min. The hydrogel was prepared by a solution casting method and the mixed solution was cast on an acrylic plate ( $55 \mathrm{~mm}$ diameter). The hydrogel was subsequently dried at 50 ${ }^{\circ} \mathrm{C}$ for $12 \mathrm{~h}$. For comparison, control hydrogels were also prepared by solution casting following the same procedure but without adding the ginger essential oil.

Scanning Electron Microscopy (SEM). The morphology of ginger fiber raw material and the cross section of the GNF hydrogel were examined using a scanning electron microscope Jeol JSM 6460LV (JEOL Ltd, Tokyo, Japan) at an acceleration voltage of 15 $\mathrm{kV}$. The samples were freeze-dried and coated with platinum using an EM ACE200 sputtering instrument (Leica, Wetzlar, Germany) prior to observation to avoid electron charging. The coating was performed in a vacuum of $6 \times 10^{-5} \mathrm{mbar}$ under a current of $100 \mathrm{~mA}$, resulting in a coating thickness of $15 \mathrm{~nm}$.

Atomic Force Microscopy (AFM). The GNF morphology and dimensions were further analyzed using a Veeco MultiMode Scanning Probe atomic force microscope (Bruker, Santa Barbara, CA) in tapping mode with a tip model TESPA (antimony (n)-doped silicon) (Bruker, Camarillo, CA). A drop of the diluted suspension (0.01 wt $\%)$ was placed on a clean mica surface and left to dry at $22-23{ }^{\circ} \mathrm{C}$. The nanofiber width was determined from the height images using the Nanoscope V software, and the average values and deviations presented here are based on 100 different measurements.

Liquid Absorption Measurement. GNF hydrogels at dry state were immersed in distilled water, PBS, and BSA $\left(50 \mathrm{~g} \mathrm{~L}^{-1}\right)$ solutions at room temperature to study the liquid absorption over time. The wet weight of the hydrogels was recorded at regular intervals over 72 h. Excess liquid was removed before weighing by gently tapping the samples on a dry tissue paper. Liquid absorption was calculated as follows

$$
\text { liquid absorption }(\%)=\left(W_{t}-W_{\mathrm{d}}\right) / W_{\mathrm{d}} \times 100
$$

where $W_{\mathrm{d}}$ denotes the initial weight of the dried sample and $W_{t}$ denotes the weight at time $t$ after immersing samples in distilled water and PBS.

Hydrogel Integrity Measurement. Hydrogel structural integrity was evaluated by measuring its weight in BSA and PBS solutions at room temperature over time. Hydrogels were incubated either in BSA or PBS solution, and the wet weight at the equilibrium state was taken as $100 \%$. The wet weight of hydrogels was measured at regular intervals, and the percentage of weight remaining was determined by the following equation

$$
\text { integrity }(\%)=W_{\mathrm{w}} / W_{\mathrm{es}} \times 100
$$

where $W_{\text {es }}$ denotes the initial weight at the equilibrium state of the sample and $W_{\mathrm{w}}$ denotes the wet weight of the sample.

Water Retention Capacity. Hydrogel samples with a grammage of $40 \mathrm{~g} \mathrm{~m}^{-2}$ were allowed to swell in distilled water until they reached equilibrium. Excess water was wiped off, and then the hydrogels were left at ambient temperature for $24 \mathrm{~h}$ and weighed at regular intervals. The water retention capacity was calculated by the following equation

$$
\text { water retention capacity }(\%)=W_{\mathrm{r}} / W_{\mathrm{es}} \times 100
$$

where $W_{\mathrm{es}}$ denotes the initial weight at the equilibrium state of the sample and $W_{\mathrm{r}}$ denotes the weight retained after the sample was left to dry.

Mechanical Testing. Compressive properties of hydrogels were analyzed using a Q800 dynamic mechanical analyzer (TA Instruments, New Castle) with the compression configuration. Samples with dimensions of $5 \times 5 \mathrm{~mm}^{2}$ and thicknesses ranging between 2 and $5 \mathrm{~mm}$ were tested in wet conditions ( $800 \%$ of swelling degree). Tests were carried out with a $1 \mathrm{mN}$ preload and a strain rate of $5 \% \mathrm{~min}^{-1}$. The compressive modulus was calculated from the slope of the initial linear region of the stress-strain curves (strain value lower than 5\%). Ten measurements were taken for each sample and averaged.

Tensile properties of the hydrogels were measured using a Shimadzu Autograph AG-X universal testing machine (Shimadzu Corp., Kyoto, Japan) equipped with a load cell of $1 \mathrm{kN}$. Tests were performed at a strain rate of $2 \mathrm{~mm} \mathrm{~min}^{-1}$ and with a gauge length of $20 \mathrm{~mm}$. The samples were in the form of strips of $6 \mathrm{~mm}$ width and 80 $\mathrm{mm}$ length and were tested in wet conditions $(800 \%$ of swelling degree for comparison under similar conditions). Ten measurements were taken for each sample and averaged. Statistical analysis at a 5\% significance level based on Levene's test was used to assess the equality of variances, and the ANOVA test was performed to compare the averages of the mechanical properties.

Antibacterial Activity Measurement. Staphylococcus aureus and Escherichia coli were streaked onto Luria Bertani (LB) agar plates and incubated at $37{ }^{\circ} \mathrm{C}$ overnight. Single bacterial colonies were picked and inoculated in $5 \mathrm{~mL}$ of $\mathrm{LB}$ broth and cultivated overnight on a shaker $(300 \mathrm{rpm})$ at $37{ }^{\circ} \mathrm{C}$. The bacterial concentration was determined by viable count, which was adjusted to correspond to $10^{9} \mathrm{CFU} \mathrm{mL} \mathrm{m}^{-1}$. The antimicrobial activity of ginger nanofibers was studied by spreading $S$. aureus and E. coli $\left(10^{7} \mathrm{CFU}\right.$ in $\left.100 \mu \mathrm{L}\right)$ on Mueller Hinton Agar and placing the hydrogels onto the bacterial lawn. The plates were incubated at $37^{\circ} \mathrm{C}$ for $20 \mathrm{~h}$ and the zones of inhibition were visualized by acquiring images at a magnification of $12.5 \times$ using an Olympus SZX9 stereo zoom microscope, (Olympus, Solna, Sweden).

Direct antimicrobial activity of the ginger material (contactdependent) was determined on Mueller Hinton Agar following addition of S. aureus and E. coli $\left(10^{5} \mathrm{CFU}\right.$ in $\left.10 \mu \mathrm{L}\right)$ on top of the membranes. The plates were incubated at $37{ }^{\circ} \mathrm{C}$ for $20 \mathrm{~h}$, followed by image acquisition. The hydrogels were placed upside down on a new Mueller Hinton plate for $10 \mathrm{~s}$ and removed to determine bacterial viability. After $20 \mathrm{~h}$ of incubation, the bacterial growth was visualized by capturing images using an Olympus SZX9 microscope at 12.5X magnification (Olympus, Solna, Sweden).

Primary Cell Isolation and Cultures. Primary skin cells were isolated from human tissue obtained from healthy patients undergoing routine reduction abdominoplasty at the University Hospital in Linköping, Sweden. All human tissue and cells were handled in accordance with guidelines postulated by Linköping University under approval from the Swedish Ethical Review Authority (no. 2018/9731). Briefly, human keratinocytes and fibroblasts were isolated by mechanical and enzymatic dissection of viable epidermis and dermis under sterile conditions. For isolation of fibroblasts, skin samples were repeatedly washed in sterile PBS and then subcutaneous fat was 

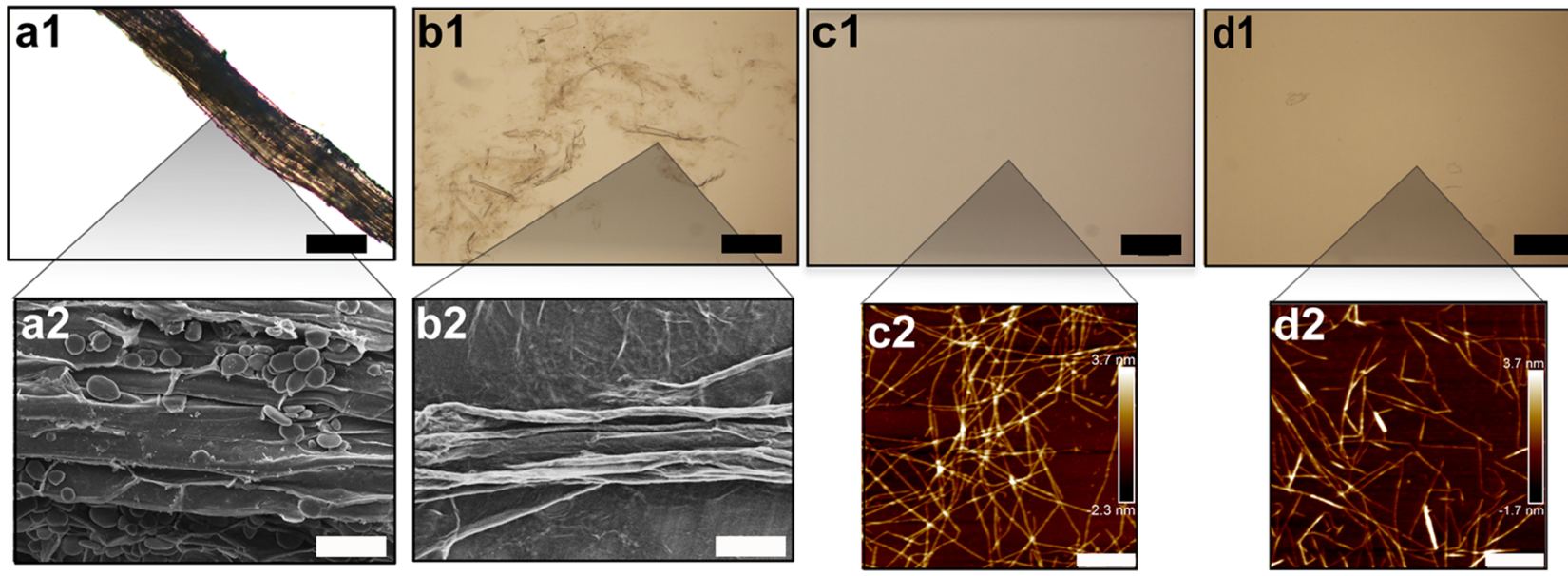

Figure 1. OMs of the ginger raw material (a1) before and (b1) after the alkali treatment step. OM images of the homogenized nanofiber suspension after (c1) only TEMPO treatment (T-GNF) and (d1) alkali and TEMPO treatment (AT 4\%-GNF) (scale bar: $200 \mu \mathrm{m})$. SEM images of dried ginger fibers: (a2) nonalkali treated and (b2) after alkali treatment (scale bar: $50 \mu \mathrm{m}$ ). AFM images of dried nanofiber suspensions (c2) TGNF and (d2) AT 4\%-GNF (scale bar: $400 \mathrm{~nm}$ ).

mechanically removed. The remaining dermis was dissected into approximately two hundred $1 \times 3 \mathrm{~mm}^{2}$ pieces, carefully avoiding epidermis and irregular structures such as large vessels, and placed in Dulbecco's modified Eagle's medium (DMEM, Gibco Thermo Fisher Scientific, Paisley, U.K.) with $165 \mathrm{U} \mathrm{mL}^{-1}$ collagenase (Gibco Thermo Fisher Scientific, Paisley, U.K.) and $2.5 \mathrm{mg} \mathrm{mL}^{-1}$ dispase (Gibco Thermo Fisher Scientific, Paisley, U.K.) and incubated at 37 ${ }^{\circ} \mathrm{C}, 5 \% \mathrm{CO}_{2}$, and $95 \%$ humidity for $12 \mathrm{~h}$. After enzymatic digestion, the tissue is mostly dissolved and so the suspension is centrifuged for $5 \mathrm{~min}$ at $200 \mathrm{~g}$ and the resulting cell pellet resuspended in fibroblast medium (DMEM containing $10 \%$ fetal calf serum, $50 \mathrm{U} \mathrm{mL}^{-1}$ penicillin, and $50 \mathrm{mg} \mathrm{mL}^{-1}$ streptomycin). This was repeated twice with a fresh medium to wash the cells. The final cell pellet was dissociated, and the cells were seeded into $75 \mathrm{~cm}^{2}$ culture flasks (Falcon, Corning Inc; Corning, NY) in fibroblast medium and kept in an incubator at $37{ }^{\circ} \mathrm{C}, 5 \% \mathrm{CO}_{2}$, and $95 \%$ humidity. The medium was changed three times per week.

For isolation of keratinocytes, samples were processed according to a modification of the protocol described by Rheinwald and Green. ${ }^{45}$ Briefly, samples were repeatedly washed in sterile PBS, and subcutaneous fat was mechanically removed. The remaining tissue was cut into $5 \times 5 \mathrm{~mm}^{2}$ pieces and placed in DMEM with $2.5 \mathrm{mg}$ $\mathrm{mL}^{-1}$ dispase at $7{ }^{\circ} \mathrm{C}$ overnight. The epidermis was then manually removed from the dermis and placed in DMEM containing $0.02 \%$ versene/0.1\% trypsin (Gibco Thermo Fisher Scientific, Paisley, U.K.) and incubated for $15 \mathrm{~min}$ at $37{ }^{\circ} \mathrm{C}, 5 \% \mathrm{CO}_{2}$, and $95 \%$ humidity. Pooled supernatants were centrifuged for $5 \mathrm{~min}$ at $365 \mathrm{~g}$ and the resulting cell pellet washed in DMEM (Gibco Thermo Fisher Scientific). Cells were seeded into $75 \mathrm{~cm}^{2}$ culture flasks (Falcon, Corning Inc) with keratinocyte serum-free medium (KSFM; containing $1 \mathrm{mg} \mathrm{L}^{-1}$ epidermal growth factor, $25 \mathrm{mg} \mathrm{L}^{-1}$ bovine pituitary extract, $50 \mathrm{U} / \mathrm{mL}$ penicillin, and $50 \mathrm{mg} / \mathrm{mL}$ streptomycin; Gibco Thermo Fisher Scientific) and kept in an incubator at $37^{\circ} \mathrm{C}$, $5 \% \mathrm{CO}_{2}$, and $95 \%$ humidity. The medium was changed three times per week.

Initial tests where cells were seeded on hydrogels were performed. Briefly, 200,000 keratinocytes or fibroblasts were seeded on $1 \times 1 \mathrm{~cm}^{2}$ hydrogels. Following $48 \mathrm{~h}$ of incubation at $37{ }^{\circ} \mathrm{C}, 5 \% \mathrm{CO}_{2}$, and $95 \%$ humidity, materials were fixed in $4 \%$ paraformaldehyde, dehydrated using an ethanol series (70, 95, and 99.5\%), and embedded in paraffin. Then, $7 \mu \mathrm{m}$ thick sections were mounted on microscopy slides and stained using AlexaFluor546-conjugated Phalloidin (Thermo Fisher Scientific) and 4',6-diamidino-2-phenylindole (Thermo Fisher Scientific) to visualize cells. Samples were examined using a BX41 light/fluorescence microscope with a DP70 CCD camera (Olympus, Stockholm, Sweden), and cell nuclei were manually counted.

Cell Viability and Proliferation. Following establishment of primary cultures, cells were enzymatically detached using $0.02 \%$ versene $/ 0.1 \%$ trypsin and seeded in six-well plates (Falcon, Corning Inc). Cells were allowed to adhere for $24 \mathrm{~h}$ and were subsequently covered with $\varnothing 10 \mathrm{~mm}$ discs of T-GNF or AT 4\%-GNF and cultured for $72 \mathrm{~h}$. Keratinocyte and fibroblast cultures in three replicate wells without addition of material served as controls. Every $24 \mathrm{~h}$, for $72 \mathrm{~h}$ in total, the cells were detached using a $0.02 \%$ versene $/ 0.1 \%$ trypsin solution at $37{ }^{\circ} \mathrm{C}$ for approximately $10 \mathrm{~min}$, stained with trypan blue to distinguish viable cells, and counted using an EVE automated cell counter (NanoEnTek, Waltham, MA). All experiments were performed in biological triplicate (separate cell cultures) and methodological duplicate (staining and cell counting). Numbers of viable cells were recorded and analyzed using Prism 8.4.2 (Graphpad LLC, LaJolla, CA). A two-way ANOVA coupled with a Holm-Sidak post-test was used to compare cell numbers over time in all groups of the same cell type, and $p<0.05$ was considered significant. Cell numbers in the treatment groups were normalized to nontreated controls and expressed as a proliferative index according to the following equation

$$
\text { proliferative index }=C_{\mathrm{T}} / C_{\mathrm{T} 0}
$$

where $C_{\mathrm{T}}$ denotes the mean number of viable cells at the analyzed time point and $C_{\mathrm{T} 0}$ denotes the mean number of viable cells at the starting time point. All values are plotted as mean \pm standard deviation (SD) unless otherwise stated.

Cell Migration Assay. To assess the effects of T-GNF or AT 4\%GNF on cell migration, fibroblasts and keratinocytes were seeded in separate triplicate six-well plates as described above. Cells were cultured until confluency, and a denuded scratch was produced using a p200 pipette tip across the center of the wells. Cultures were covered with the hydrogels and examined at $0,24,48$, and $72 \mathrm{~h}$ using an IX51 light microscope with a DP70 CCD camera (Olympus, Stockholm, Sweden). Photos were captured at $10 \times$ magnification and analyzed using Image $1.52 \mathrm{p}(\mathrm{NIH})$ to measure the remaining cellfree area in the denuded streak. The areas were normalized to the area at time 0 according to the following equation

$$
\text { scratch closure }(\%)=A_{\mathrm{t}} / A_{\text {start }} \times 100
$$

where $A_{\mathrm{t}}$ denotes the mean remaining denuded area at the experimental time and $A_{\text {Start }}$ denotes the initial mean wound area. A mixed-effect restricted maximum likelihood (REML) model coupled with a Holm-Sidak post-test was used to compare cell numbers over time in all groups of the same cell type, and $p<0.05$ was considered 
significant. All values are plotted as mean \pm standard deviation (SD) unless otherwise stated.

\section{RESULTS AND DISCUSSION}

Production and Characterization of Ginger Nanofibers. Hydrogels with different liquid absorption capacities are interesting for wound dressing applications. Different types of wounds and different stages of the healing process may have different requirements for an optimal dressing. The process for material preparation can result in different material properties, and natural starting materials can have different advantageous properties. We sought to leverage this knowledge to develop a multifunctional material aimed at advanced wound dressings, sourced and produced with sustainability in mind. Ginger nanofibers were produced by TEMPO oxidation combined with high-pressure homogenization before and after alkali treatment. The effects of the different processing routes and the nanofiber compositional varieties derived from the alkali treatments were further studied with regard to hydrogel properties (Figure 1). The chemical composition without alkali treatment was $25.1 \pm 0.5 \% \quad \alpha$-cellulose, $40.5 \pm 0.6 \%$ hemicellulose, $5.5 \pm 0.9 \%$ Klason lignin, and $5.1 \pm 1.3 \%$ extractives. These results are in agreement with those of Abral et al., who found cellulose and hemicellulose contents to be 30 and 44\%, respectively. ${ }^{35}$ However, Zaki, Abdullah, and Ahmad reported a higher value of cellulose $(65.2 \%)$ and a lower hemicellulose value $(10.1 \%){ }^{46}$ The differences in proportions of each individual component can be explained by a number of factors related to the analysis methodology or the sample itself. These factors include country of origin, harvesting conditions, industrial processing, and whether the ginger is fresh, dried, or processed. ${ }^{47}$ Besides the high contents of cellulose and hemicellulose, ginger has a significant amount of starch varying between 30 and $60 \% .^{48,49}$ It is generally accepted that alkali treatment changes the composition of the fibers by targeting the noncellulosic components, such as starch, hemicellulose, and pectin. ${ }^{50}$ Alkali treatment was applied to ginger fibers before TEMPO oxidation, aiming to reduce the hemicellulose content and alter the characteristics of the nanofibers. This directly influenced the properties of the hydrogels prepared with them. The content of hemicellulose after oxidation was $11.0 \pm 0.8 \%$, which supports that the alkali treatment reduces noncellulosic components. The process yields, with and without alkali-treated ginger as feedstock, are presented in Table 1.

Table 1. Total Yield and Weight after Each Step of the Ginger Nanofiber Production Process

\begin{tabular}{ccccc} 
& $\begin{array}{c}\text { initial } \\
\text { weight } \\
{[\mathrm{g}]}\end{array}$ & $\begin{array}{c}\text { weight after } \\
\text { weight after alkali } \\
\text { treatment }[\mathrm{g}]\end{array}$ & $\begin{array}{c}\text { TEMPO oxidation } \\
{[\mathrm{g}]}\end{array}$ & $\begin{array}{c}\text { total } \\
\text { yield } \\
{[\%]}\end{array}$ \\
T-GNF & 10 & & 4.3 & 43.3 \\
AT 4\%- & 10 & 1.5 & 0.6 & 6.4 \\
GNF & & & & \\
\hline
\end{tabular}

The lower yield (6.4\%) obtained using alkali-treated ginger is attributed to the material loss during the alkali step. However, this value is higher than the ginger nanofiber yield of $3.1 \%$ reported by Jacob et al. who also applied an alkali treatment on ginger fibers under similar conditions to reduce the noncellulosic components. ${ }^{32}$

The optical micrographs (OMs) are presented in Figure 1, showing the raw material, the ginger fiber with and without alkali treatment, and GNFs, together with atomic force microscopy (AFM) height images (Figure 1c2,d2).

From Figure 1c1, it is possible to note that alkali treatment generated microsized structures composed of fragments of parenchyma cells and fibers of different sizes. The representative SEM images before and after alkali treatment are shown in Figure $1 \mathrm{a} 2$ and b2, respectively. Starch granules can be observed with an oval shape and a smooth surface without fissures, typically associated with fibers in ginger. ${ }^{49,51} \mathrm{~A}$ comparison between Figure $1 \mathrm{a} 2$ and $\mathrm{b} 2$ indicates that besides reducing the hemicellulose content, the alkali treatment also reduced the starch content since the starch granules are absent in the alkali-treated fibers. Furthermore, the OMs of the GNF suspensions obtained from TEMPO oxidation directly followed by homogenization and after alkali treatment of ginger are presented in Figure $1 \mathrm{cl}$ and d1, respectively. In both conditions, it was not possible to observe any large structures after the TEMPO treatment confirming that their size was reduced to the nanoscale.

The overview AFM images of GNFs together with their size distribution (Figure S1) are provided in the Supporting Information. The size of the GNFs was measured from AFM height images (Figure $1 \mathrm{c} 2, \mathrm{~d} 2$ ) and the averaged width values of T-GNFs and AT 4\%-GNFs were $1.7 \pm 0.7$ and $2.0 \pm 1.1$ $\mathrm{nm}$, respectively. Overall, it can be noted that the widths were comparable for both samples (Figure S1, Supporting Information). Additionally, the ginger nanofiber width is in agreement with the values of TEMPO nanofibers isolated from wood reported elsewhere. ${ }^{44,52}$

Liquid Absorption Capacity of Ginger Nanofiber Hydrogels. One of the most important properties of hydrogels for wound dressing applications is the water/liquid absorption, sometimes expressed as the swelling degree, which directly reflects the hydration ability. ${ }^{53}$ A moist environment allows for cell migration of epithelial cells and leukocytes, and distribution of growth factors, into the wound bed, thus facilitating the wound healing process. ${ }^{54}$

The liquid absorption over time of hydrogels prepared from GNFs isolated directly after TEMPO treatment (T-GNF) and alkali treatment using 2 wt \% (AT 2\%-GNF) and 4 wt \% (AT $4 \%$-GNF) sodium hydroxide is shown in Figure 2.

The highest water absorption capacity was obtained by the T-GNF hydrogel ( $6200 \%)$, which was about five times higher than the value obtained by the AT $4 \%$-GNF hydrogel $(\sim 1350 \%)$, as well as that measured for the reference hydrogel prepared from wood-based nanofibers $(\sim 1250 \%)$ (Figure $2 \mathrm{a} 1)$. From Figure 2a2, it is evident that the T-GNF hydrogel reached the same absorption capacity value as that of AT 4\%GNF at equilibrium in the first few minutes after immersion in water. It is worth highlighting this because the T-GNF hydrogel showed water absorption capacity of 62 times its dry weight, owing to which T-GNF can be compared to a superabsorbent hydrogel. ${ }^{55-57}$ This is an interesting result as the hydrogel was prepared only from natural polymer without using any cross-linker, swelling agent, or highly hydrophilic synthetic polymer and through a simple methodology. The high water absorption of T-GNF is likely associated with the natural composition of ginger that is rich in both hemicellulose and starch in contrast with AT 4\%-GNF and the wood-based reference material, where these components are reduced (and in the case of wood, the starch content is absent). Zhang et al. prepared a superabsorbent hydrogel based on the high swelling capacity of hemicellulose. ${ }^{58}$ Hydrophilic polymers, such as 

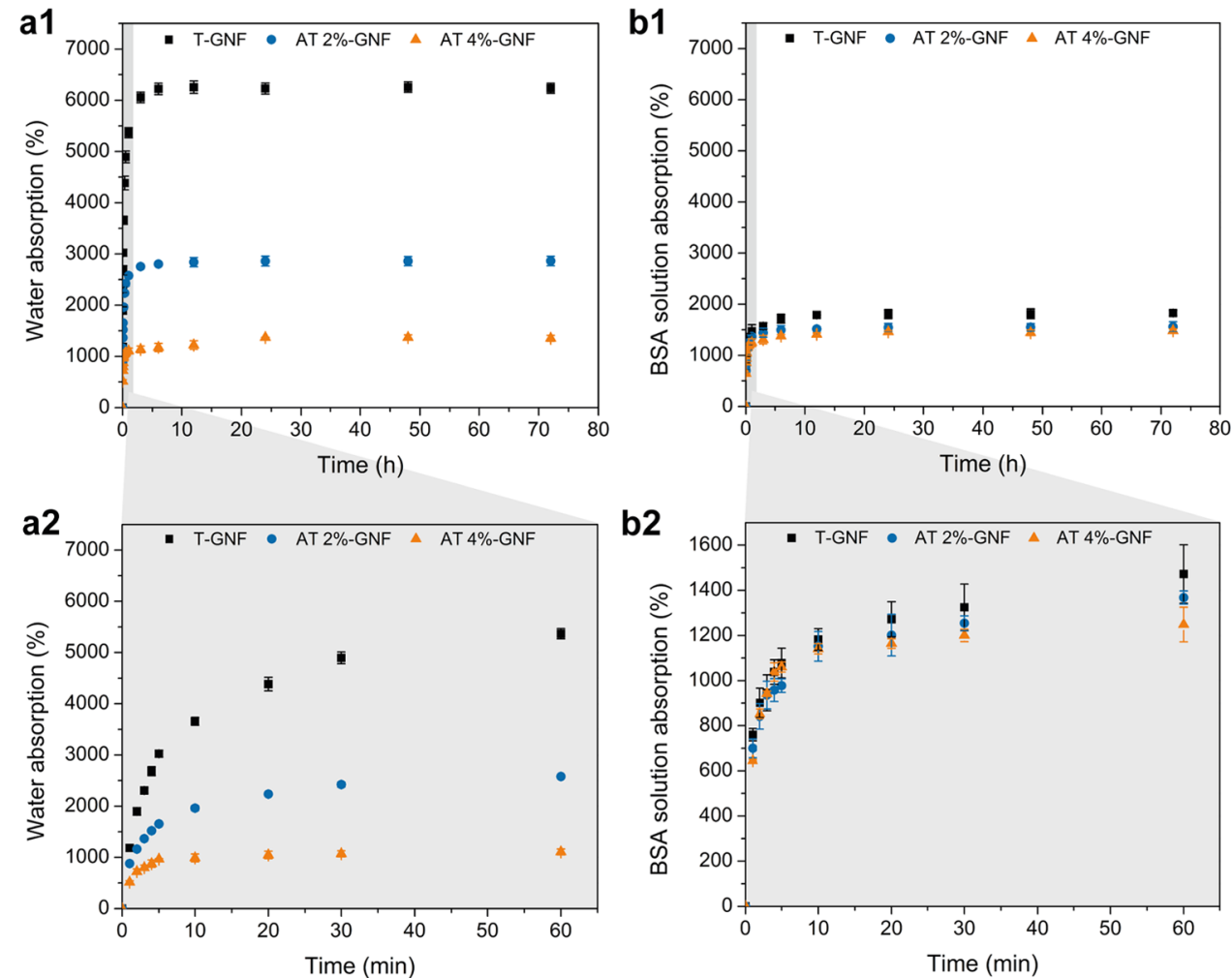

Figure 2. Liquid absorption of the hydrogels $\left(40 \mathrm{~g} \mathrm{~m}^{-2}\right)$ in (a1) water and (b1) BSA solution. Liquid absorption of hydrogel curves expanded for the first hour in (a2) water and (b2) BSA solution.

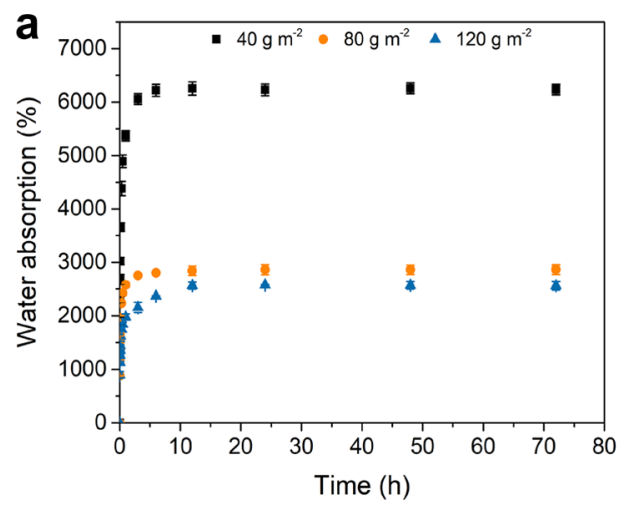

b

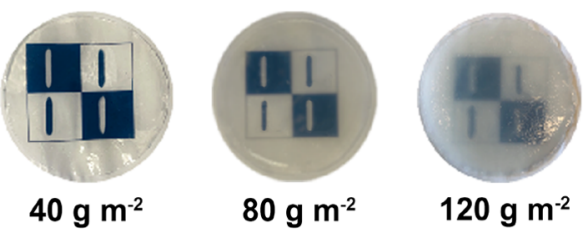

Figure 3. (a) Water absorption as a function of time for T-GNF hydrogels at different grammages. (b) Photographs of the T-GNF hydrogels at different grammages after the equilibrium swelling degree was reached.

starch, carrageenan, and poly-acrylamide, are commonly added to hydrogels to enhance their swelling capacity. ${ }^{59}$ These results suggest that the natural composition of ginger can be beneficial for the preparation of hydrogels with a high water absorption capacity. Additionally, alkali treatments can be used to effectively alter the liquid absorption capacity of the hydrogels by modifying the chemical composition of the raw materials.

The swelling behavior was also evaluated in BSA solution to more closely resemble the wound environment in terms of a higher abundance of proteins (Figure 2b1,b2). The difference between the hydrogel liquid absorption capacities was less pronounced in BSA solution, and the same behavior was also observed for PBS solution (Figure S2, Supporting Information). However, the T-GNF hydrogel still showed the highest swelling degree at equilibrium when compared with AT $2 \%$ GNF and AT 4\%-GNF hydrogels. The swelling at equilibrium was overall lower in BSA than in water, which could be attributed to the repulsive electrostatic interactions between the BSA molecules and the hydrogels. The swelling behavior of neutral hydrogels is controlled by opposing forces resulting from the spontaneous mixing of the fluid molecules with the polymer chains and the retractive forces developed inside the structure. The equilibrium swelling state is reached when these forces are balanced. The presence of ionic groups in the hydrogel structure and ions in the surrounding media generate additional forces that affect the balance, thus influencing the liquid absorption properties. ${ }^{60}$ Both hydrogels are negatively charged as indicated by the negative $\zeta$-potentials: $-44.8 \pm 0.7$ $\mathrm{mV}$ for T-GNF and $-65.6 \pm 0.7 \mathrm{mV}$ for AT $4 \%$-GNF. All proteins contain positively and negatively charged groups, and their effective charge is a result of the balance of these groups at a given $\mathrm{pH}$. The BSA solution was prepared in water, and 

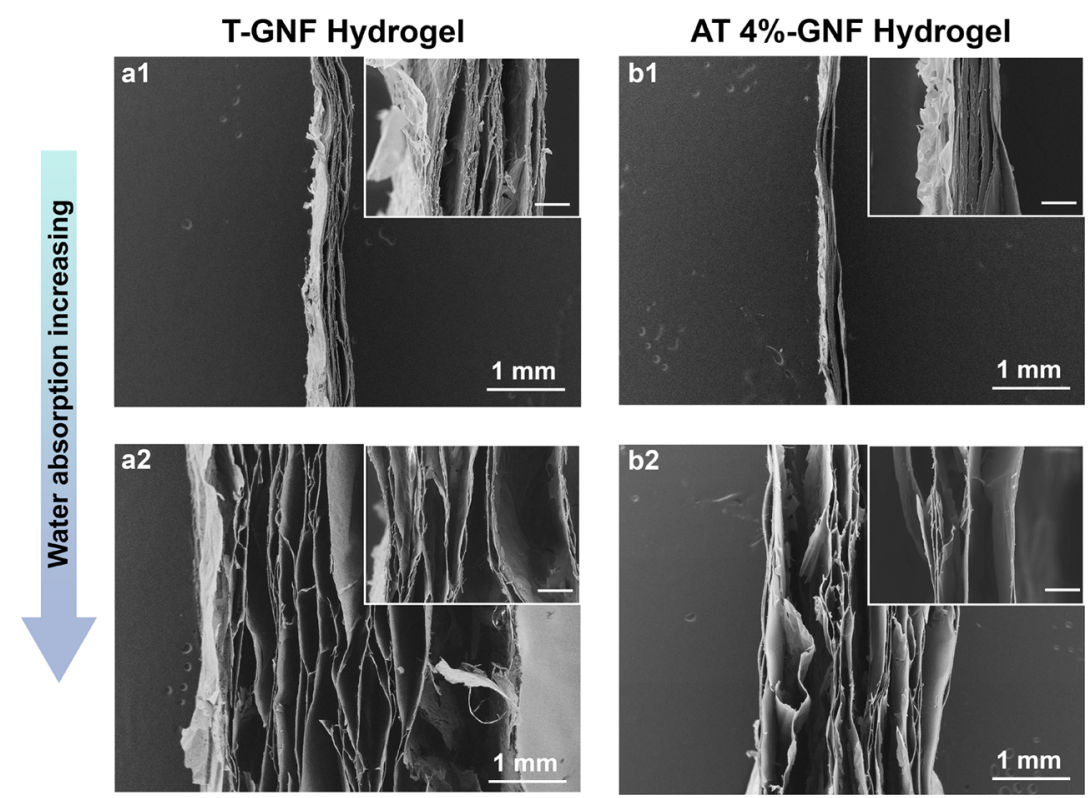

Figure 4. SEM images of ginger nanofiber hydrogels of $120 \mathrm{~g} \mathrm{~m}^{-2}$ grammage showing the swelling mechanism due to water absorption. (a1) TGNF and (b1) AT 4\%-GNF at $800 \%$ of swelling degree and (a2) T-GNF and (b2) AT 4\%-GNF at equilibrium swelling degree. Scale bar of the insets: $100 \mu \mathrm{m}$.

the effective charge value at $\mathrm{pH}$ higher than the isoelectric point of BSA $(4.7)^{61}$ has been reported to be around -7.0 $\mathrm{mV} .^{62}$ Thus, the ionic interactions between the carboxyl groups on the GNF surface with charged groups in the BSA molecules might have counteracted the swelling of the hydrogel structure, resulting in decreased liquid absorption. Similarly, Kono et al. reported a reduction of the swelling degree due to the repulsive electrostatic interactions between the BSA molecules and the hydrogel that had carboxylate anions as the predominant charged species. ${ }^{63}$

The structural integrity of the hydrogels was evaluated as the weight remaining after immersing the samples either in BSA or in PBS solution (Tables S1 and S2, Supporting Information). Within 40 days, the wet weight remained constant for all of the hydrogels, indicating no material loss in either incubation condition. It is noteworthy that the hydrogels maintained their structural integrity for long periods of time. The water retention capacity reflects the ability of a dressing material to hold water molecules within its structure, which may be important for keeping the wound moist. The water retention capacities of T-GNF $(16 \pm 3.6 \%)$ and AT 4\%-GNF (12 \pm $3.3 \%)$ hydrogels were comparable, after exposure to air, at room temperature for $24 \mathrm{~h}$ (Figure S3, Supporting Information).

Since the T-GNF hydrogel showed a higher liquid absorption capacity, the influence of the hydrogel grammage on water absorption was investigated and is presented in Figure 3.

From Figure 3, it is noted that the highest swelling degree was reached by the hydrogel with the lowest grammage ( $40 \mathrm{~g}$ $\mathrm{m}^{-2}$ ). The increase in the grammage led to a significant decrease in swelling. This behavior can be due to a higher degree of nanofibers, which favors the formation of connected points, and, in turn, increases the rigidity of the network and reduces the swelling capacity. It should be noted that controlling the hydrogel grammage could be another approach to adjust the liquid absorption capacity. Comparing the hydrogels with different grammages (Figure $3 \mathrm{~b}$ ), it is possible to observe that the transparency reduced as the grammage increased. Thus, besides the highest swelling degree, the hydrogel produced with T-GNF at $40 \mathrm{~g} \mathrm{~m}^{-2}$ presented good transparency, which is advantageous for dressing materials as it allows for monitoring of the wound during the healing process (Figure S4, Supporting Information).

The morphology of the hydrogels produced with T-GNFs and AT 4\%-GNFs at a low swelling degree (800\%) and after they reached the equilibrium swelling degree in water was investigated using SEM and is presented in Figure 4.

The cross sections of both GNF hydrogels displayed a layerlike structure after vacuum-assisted filtration assembly (Figure 4). The layered networks are formed as the water evaporates via fiber-fiber bonding due to secondary attraction forces, including hydrogen bonds, that develop between the nanofibers. ${ }^{64}$ This layered nanofiber network structure has been documented by other studies in the literature. ${ }^{65-69}$ Comparing the different conditions shown in Figure 4, it is possible to observe the swelling mechanism in which the layers are more spaced out with increasing water absorption. Additionally, it can be noted that the T-GNF hydrogel (Figure 4a2) is thicker than the AT 4\%-GNF hydrogel (Figure 4b2) at the same swelling degree, which corroborates the higher liquid absorption capacity of the former.

Mechanical Properties. The mechanical properties of the hydrogels and the representative tensile and compressive stress-strain curves are displayed in Figure 5 and Table S3 (Supporting Information). The initial linear region of the stress-strain curves used to calculate the compressive modulus is provided in Figure S5 (Supporting Information). From Figure 5, it can be noted that the T-GNF hydrogel exhibited better compressive properties, displaying a compressive modulus of $77.5 \pm 3.6 \mathrm{kPa}$, which is more than two times the value obtained for the AT 4\%-GNF hydrogel $(33.7 \pm 4.7$ $\mathrm{kPa})$. It has been demonstrated that hemicellulose facilitates the nanofibrillation of wood and contributes to enhanced stiffness and strength of composites. ${ }^{70}$ Even though the length of nanofibers cannot be determined from AFM images, due to 
a

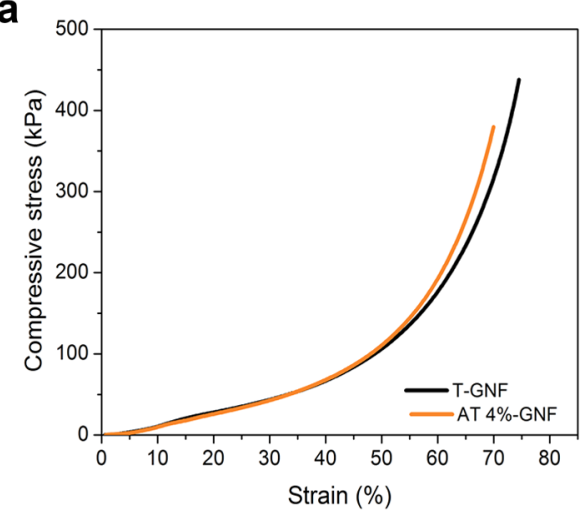

C

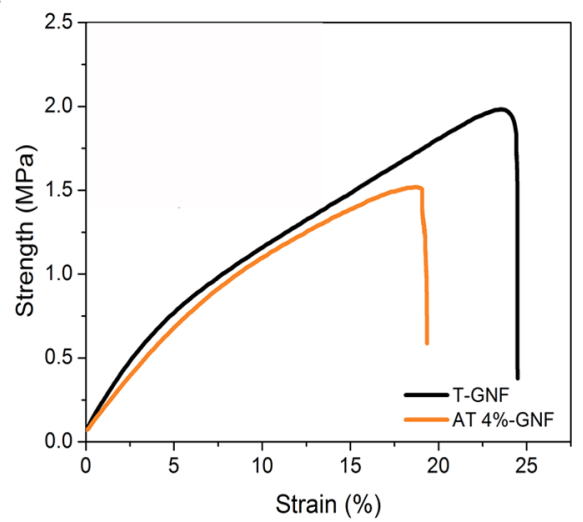

b

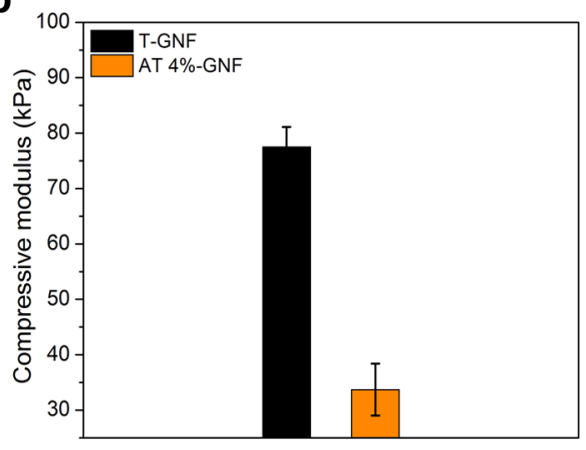

d

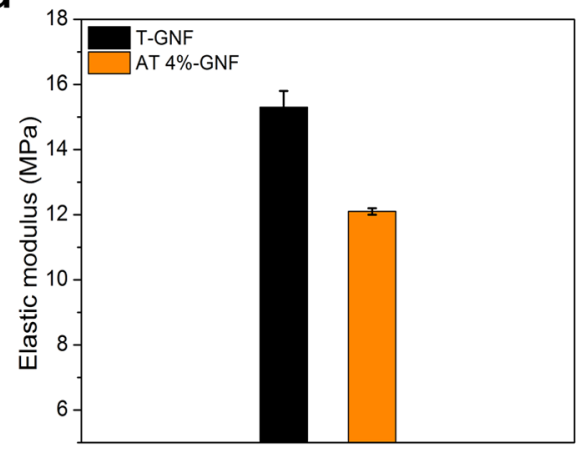

e

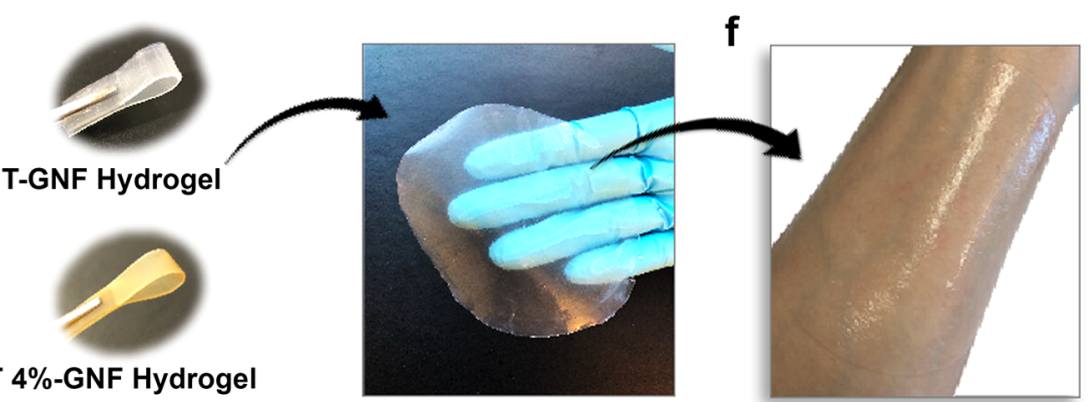

Figure 5. Mechanical properties and hydrogel appearance. (a) Representative compressive stress-strain curves and (b) the compressive modulus of the GNF hydrogels at $40 \mathrm{~g} \mathrm{~m}^{-2}$ determined in wet conditions. (c) Representative tensile stress-strain curves and (d) tensile properties of the GNF hydrogels at $40 \mathrm{~g} \mathrm{~m}^{-2}$ determined in wet conditions. (e) Photographs of the T-GNF and AT 4\%-GNF hydrogels and (f) application of the TGNF hydrogel on the forearm.

entanglements, the T-GNFs (Figure 1c2) appear to have been isolated with a preserved length that allows for increased interconnectivity compared to AT 4\%-GNFs (Figure 1d2), which showed shorter nanofibers. Longer nanofibers with a high aspect ratio result in more entanglements and contact points, leading to stronger networks. ${ }^{66}$ Tensile properties such as the strength and strain to failure are important for wound dressings as the material should not break during the fixation process, wearing, or removal. ${ }^{71}$

From Figure 5c,d, The T-GNF hydrogel exhibited excellent mechanical performance with a tensile strength of $2.1 \pm 0.2$ $\mathrm{MPa}$ and an elastic modulus of $15.3 \pm 0.3 \mathrm{MPa}$, compared to the AT 4\%-GNF hydrogel with a tensile strength of $1.6 \pm 0.1$ $\mathrm{MPa}$ and an elastic modulus of $12.1 \pm 0.1 \mathrm{MPa}$. Sun et al. reported elastic modulus in the range of $0.4-3.0 \mathrm{MPa}$ and tensile strength less than $0.25 \mathrm{MPa}$ measured in wet conditions of the TEMPO-oxidized cellulose nanofiber films obtained by solvent casting. ${ }^{72}$ These values were lower than those obtained for GNFs, and the difference might be associated with the method used for cellulose nanofiber processing, which affects the properties (e.g., mechanical, optical) of the resulting materials. ${ }^{73}$ Studies in the literature comparing the effects of different processes, such as casting and filtration, on the mechanical properties have reported that the filtration technique leads to a mechanically stronger nanofiber network relative to the casting method. ${ }^{74-76}$ According to Sehaqui et al., the network properties are very sensitive to nanofiber orientation, and the higher modulus and tensile strength are likely attributed to the increased in-plane orientation of the nanofibers. $^{74}$

Moreover, the elastic modulus of skin reported in the literature varies considerably depending on the type and conditions of the mechanical test, and for the tensile test, it ranges from 4.6 and $20 \mathrm{MPa}^{77}$ Since the values reported here are within this range, hydrogels possess the potential for wound dressing applications. Additionally, the hydrogels 


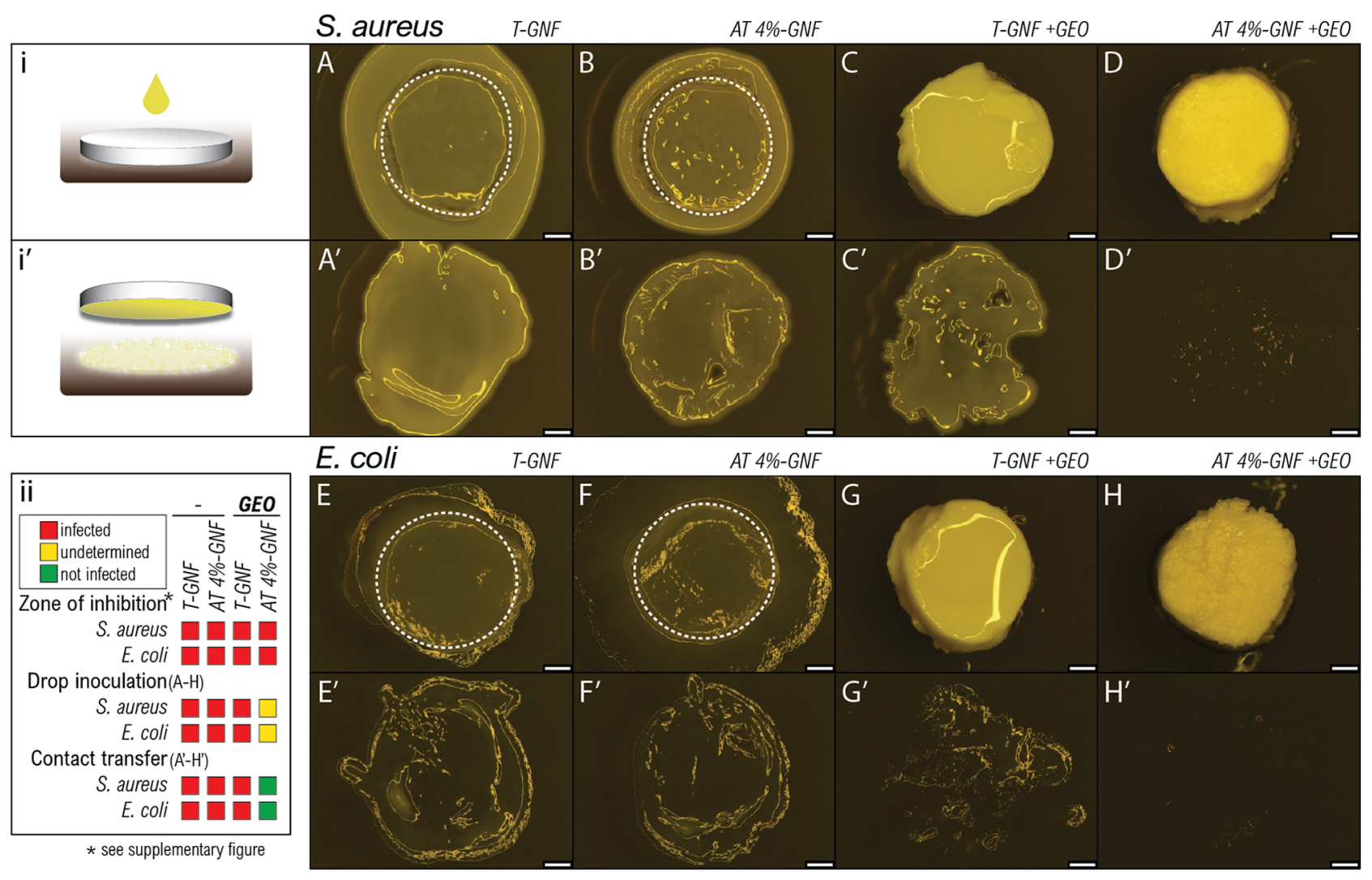

Figure 6. Images from microbiological assays against S. aureus and E. coli for T-GNF and AT 4\%-GNF hydrogels before and after functionalization with ginger essential oil (GEO). Method sketches of (i) drop inoculation of hydrogels (upper rows: A-H) and (i') subsequent contact-dependent transfer of microbes to fresh agar (lower rows: $\left.\mathrm{A}^{\prime}-\mathrm{H}^{\prime}\right)$. (A-D) S. aureus-infected hydrogels and $\left(\mathrm{A}^{\prime}-\mathrm{D}^{\prime}\right)$ contact transfer of $S$. aureus and $(\mathrm{E}-\mathrm{H})$ E. coli-infected hydrogels and $\left(\mathrm{E}^{\prime}-\mathrm{H}^{\prime}\right)$ contact transfer of $E$. coli. Panel (ii) shows the summary of antimicrobial results. * zone of inhibition assay is shown in Supporting Information S6. Dashed lines represent the approximate area of hydrogel beneath the bacterial colony; scale bars, $1 \mathrm{~mm}$.

presented good flexibility (Figure 5e) and shape retention and were gently pliant to the skin (Figure 5f).

Antibacterial Effects. To evaluate the antibacterial activity of hydrogels, microbiological assays were performed against Gram-positive S. aureus and Gram-negative E. coli (Figure 6), and the overview images and the zone of inhibition are provided in Figure S6, Supporting Information.

Ginger essential oil (GEO) was chosen to functionalize the hydrogels since it has been reported to have antimicrobial activity. ${ }^{29}$ Before and after functionalization, no clear zone of inhibition could be observed for T-GNF and AT 4\%-GNF hydrogels (Figure S6), indicating no measurable inhibitory activity against $S$. aureus and $E$. coli. In contrast, Abral et al. and Jacob et al. reported good antimicrobial performance of ginger nanofiber films against different bacteria species. ${ }^{33,35}$ There are many factors affecting the antibacterial activity of ginger, such as processing methods, the type of microorganisms, ${ }^{35}$ and/or raw material storage conditions. ${ }^{78}$ Plausible reasons for the loss of antibacterial activity of the ginger nanofiber hydrogels in this study, compared to previously reported studies, could be differences in nanofiber production methods or the use of raw material stored for longer time periods as ginger is not grown in Sweden. ${ }^{31,33}$ Bacterial growth could not be determined after drop inoculation of hydrogels (Figure $6 \mathrm{~A}-\mathrm{H}$ ). However, the subsequent contact-dependent transfer of microbes to fresh agar (Figure $6 \mathrm{~A}^{\prime}-\mathrm{H}^{\prime}$ ) revealed a bactericidal effect after GEO functionalization, indicated by the absence of bacterial growth for AT 4\%-GNF (Figure $6 \mathrm{D}^{\prime}, \mathrm{H}^{\prime}$ ), compared to the bacterial growth pattern found in the other materials (Figure $6 \mathrm{~A}^{\prime}-$ $\left.\mathrm{C}^{\prime}, \mathrm{E}^{\prime}-\mathrm{G}^{\prime}\right)$.

Variation in the chemical composition of the hydrogels might explain their different antibacterial activity. Overall, GEO appears to contribute to antimicrobial hydrogels, hindering bacterial growth on the hydrogels, without releasing active antimicrobial substances under these conditions. Infected wounds are commonly treated with antibiotics, and the rising problem of antibiotic resistance is alarming. The use of completely biobased resources may be a safer way to obtain advanced wound dressing material with antimicrobial properties while minimizing cytotoxic side effects. ${ }^{79-81}$ The use of GEO for antimicrobial activity enhancement of completely ginger-based hydrogels demonstrates the potential to utilize multiple intrinsic properties of raw material to develop functional materials.

In Vitro Cytocompatibility Study. The potential use of the GNF hydrogels for wound dressing application requires an initial assessment of cytocompatibility. Since wound healing is primarily executed by the proliferation and migration of keratinocytes and fibroblasts, the migratory and proliferative behavior of primary keratinocytes and fibroblasts from human abdominal skin were separately evaluated in the presence of the T-GNF and AT 4\%-GNF hydrogels by culturing them in direct contact with the two GNF hydrogels. Initial tests where cells were seeded on top of the hydrogels revealed a low degree of adhesion with no increase in cell numbers over time (Supporting Information S7). This finding indicates that the hydrogels are unsuitable for use as cell-loaded scaffold material 
a1

Fibroblasts

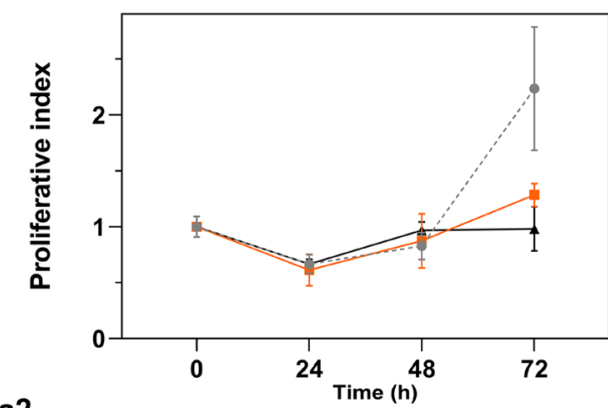

a2

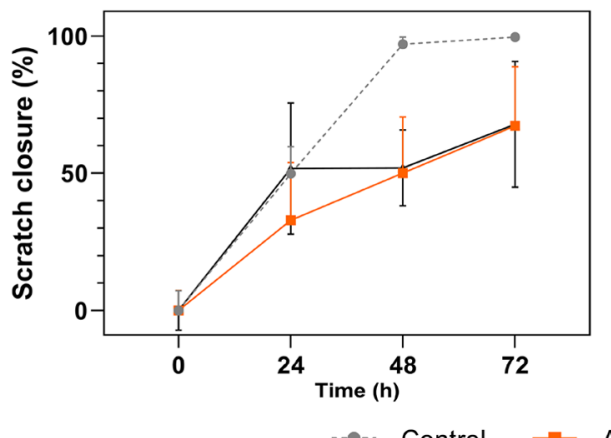

b1
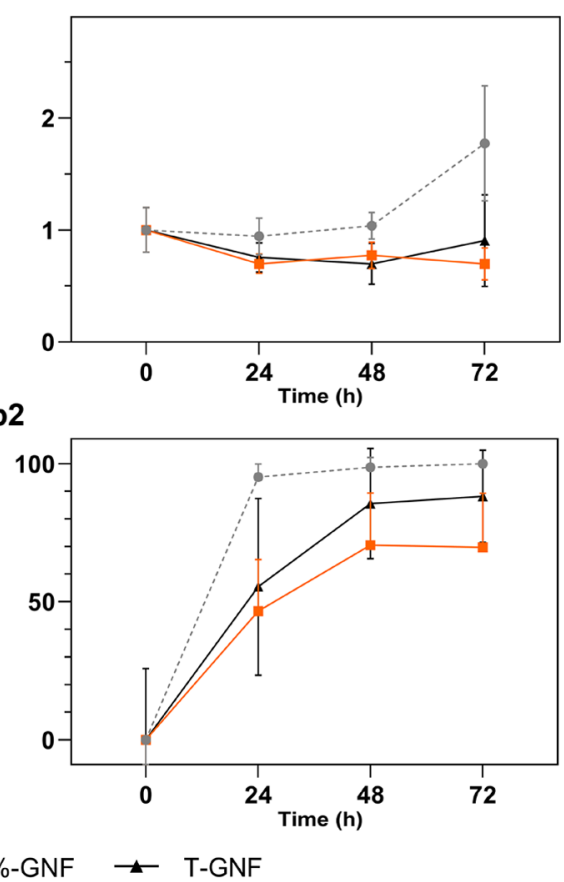

Figure 7. Effects of T-GNF and AT 4\%-GNF hydrogels on fibroblast (a1) proliferation and (a2) migration and keratinocyte (b1) proliferation and (b2) migration. $n=4$.

but suggests desirable properties for dressing applications, where incorporation of tissue from the healing wound may cause difficulties in dressing changes. A proliferation and viability assay where viable cells were counted over time and a migration assay (scratch wound repopulation) were employed for the analysis of the cell cultures, and the results are presented in Figure 7.

The proliferative index of keratinocytes and fibroblasts over time is shown in Figure $7 \mathrm{a} 1, \mathrm{~b} 1$. The interpretation of the statistical analysis revealed that both time $(49 \%, p<0.0001)$ and treatment $(7.8 \%, p<0.05)$ significantly contribute to the total variation of the proliferative index. Neither T-GNF nor AT 4\%-GNF hydrogels affected fibroblast or keratinocyte proliferation before the $72 \mathrm{~h}$ time point. Proliferation of fibroblasts was significantly impaired after $72 \mathrm{~h}$ (Figure $7 \mathrm{al} ; p$ $<0.05$ ), with a proliferative index of $0.98 \pm 0.20$ for T-GNF, $1.28 \pm 0.10$ for AT $4 \%-G N F$, and $2.23 \pm 0.55$ for the control. Proliferation of keratinocytes was significantly impaired after $72 \mathrm{~h}$ (Figure $7 \mathrm{~b} 1 ; p<0.05$ ), with a proliferative index of 0.91 \pm 0.41 for T-GNF, $0.70 \pm 0.14$ for AT $4 \%$-GNF, and $1.77 \pm$ 0.51 for the control (Figure 7a1). Migration of fibroblasts and keratinocytes over time is shown in Figure 7a2,b2. Overall, significant differences were seen for both time and treatment $(p<0.0001)$. For fibroblasts, the scratch closure of the T-GNF group was significantly lower compared to that of the control at $48 \mathrm{~h}(52 \pm 14$ vs $97 \pm 2.6 \%, p<0.005)$. The AT $4 \%-G N F$ group was significantly reduced at $48 \mathrm{~h}(50 \pm 20$ vs $97 \pm 2.6 \%$, $p<0.001)$ and $72 \mathrm{~h}(67 \pm 22$ vs $100 \pm 0.9 \%, p<0.05)$. For keratinocytes, the scratch closure for the T-GNF group was significantly reduced at $24 \mathrm{~h}(55 \pm 32$ vs $95 \pm 4.8 \%, p<0.005)$ and $72 \mathrm{~h}(88 \pm 17$ vs $100 \pm 0 \%, p<0.05)$. For AT $4 \%$-GNF, the scratch closure was significantly reduced at $24 \mathrm{~h}(47 \pm 19$ vs $95 \pm 4.8 \%, p<0.0001)$, 48 h $(71 \pm 19$ vs $99 \pm 3.6 \%, p<$ $0.001)$, and $72 \mathrm{~h}(70 \pm 20$ vs $100 \pm 0 \%, p<0.001)$. The method of placing the GNF hydrogels on top of the cells can itself interfere with normal culture conditions. ${ }^{82}$ The added barrier created by the GNF may interfere with the oxygen diffusion rate and the turnover of soluble factors near the cells. In an in vivo situation, the skin is fed by nutrients from the vascularized underlying tissue but the in vitro model is limited by free diffusion of oxygen through, and soluble factors added into, the culture medium. This may explain diminished migration and proliferation and help explain some of the limits to scratch closure in the migration assay. In light of these results, the control group without hydrogels can be considered evidence of a viable and healthy primary cell population, but comparisons between results with and without hydrogels should be carefully employed. More interesting are any differences between the GNF hydrogels and the overall viability and migratory capacity of the cells. Overall, the GNF hydrogels did not display cytotoxicity (Figure 7; Figures S8 and S9 Supporting Information). However, proliferation and migration of keratinocytes and fibroblasts were not increased; instead, the covering of culture resulted in somewhat decreased cell numbers. Wound healing is a complex process, and further evaluation both in vitro and in vivo would be imperative in future studies to establish the suitability of GNF hydrogels as wound dressings and better understand the effects of T-GNF and AT 4\%-GNF hydrogels on healing.

\section{CONCLUSIONS}

Hydrogels based on TEMPO-oxidized ginger nanofibers were successfully prepared via vacuum-assisted filtration without any cross-linker and with advantageous properties for wound dressing applications. It was shown that the liquid absorption capacity of the hydrogels could be adjusted by altering the chemical composition of ginger fibers with alkali treatment prior to nanofiber isolation and subsequent hydrogel formation. Furthermore, the grammage of the hydrogels was 
shown to dictate the absorption capacity. The hydrogel produced with ginger without alkali treatment ( $T$-GNF hydrogel) of $40 \mathrm{~g} \mathrm{~m}^{-2}$ grammage showed the highest water absorption of 62 times its initial weight and reached a value that was five times higher than the one obtained with the reference wood nanofiber hydrogel. A high swelling capacity was achieved by preserving the noncellulosic components such as starch and hemicellulose naturally found in ginger when preparing the nanofibers and their hydrogels. Additionally, the T-GNF hydrogels showed good mechanical properties with tensile strength of $2.1 \pm 0.2 \mathrm{MPa}$ and elastic modulus of 15.3 $\pm 0.3 \mathrm{MPa}$. The antimicrobial activity of ginger was not preserved after nanofiber separation, as observed from microbiological assays. However, functionalization using ginger essential oil improved antimicrobial performance against $S$. aureus and E. coli, and the absence of bacterial growth suggests that the AT 4\%-GNF hydrogel was bactericidal. However, additional experiments are needed to better understand and optimize the functionalization of ginger nanofiber hydrogels using essential oil. Cytocompatibility evaluation showed that T-GNF and AT 4\%-GNF hydrogels did not significantly affect fibroblast proliferation. Meanwhile, migration of keratinocytes was more beneficial when in contact with the T-GNF hydrogel than AT 4\%-GNF.

The current study highlights an upscalable environmentally friendly way to prepare completely ginger-based nanofiber hydrogels that combine functions attractive for wound dressing, such as tunable absorption, flexibility, and transparency, while being nontoxic and mechanically stable in moist conditions. Moreover, potential additional functionalization could further be explored with the aim of improving wound healing.

\section{ASSOCIATED CONTENT}

\section{(s) Supporting Information}

The Supporting Information is available free of charge at https://pubs.acs.org/doi/10.1021/acs.biomac.1c00215.

AFM images and size distribution of the nanofibers obtained with and without alkali-treated ginger; PBS solution absorption of the T-GNF and AT 4\%-GNF hydrogels; hydrogel integrity in BSA and PBS solutions; water retention of the T-GNF and AT 4\%-GNF hydrogels; photographs of the T-GNF hydrogels at 40 $\mathrm{g} \mathrm{m}^{-2}$ grammage after reaching the equilibrium state; representative compressive stress-strain curves enlarged at $30 \%$ strain; compilation of the hydrogel mechanical properties; microbiological assays for T-GNF and AT 4\%-GNF hydrogels before and after functionalization with ginger essential oil (overview and inhibition zones); investigation of cell attachment to the T-GNF hydrogel; and images from scratch assay showing migration of fibroblasts and keratinocytes at different time points (PDF)

T-GNF hydrogel applied on a forearm (AVI)

\section{AUTHOR INFORMATION}

\section{Corresponding Author}

Linn Berglund - Division of Materials Science, Department of Engineering Sciences and Mathematics, Luleå University of Technology, SE-971 87 Luleå, Sweden; (1) orcid.org/00000002-6247-5963; Phone: +46 (0)920 493381;

Email: linn.berglund@ltu.se

\section{Authors}

Paula Squinca - Division of Materials Science, Department of Engineering Sciences and Mathematics, Luleå University of Technology, SE-971 87 Luleå, Sweden; Embrapa Instrumentation, 13561-206 São Carlos, SP, Brazil; Graduate Program of Chemical Engineering, Federal University of São Carlos, 13565-905 São Carlos, SP, Brazil

Kristina Hanna - Center for Disaster Medicine and Traumatology, Department of Biomedical and Clinical Sciences, Linköping University, SE-581 85 Linköping, Sweden

Jonathan Rakar - Center for Disaster Medicine and Traumatology, Department of Biomedical and Clinical Sciences, Linköping University, SE-581 85 Linköping, Sweden

Johan Junker - Center for Disaster Medicine and Traumatology, Department of Biomedical and Clinical Sciences, Linköping University, SE-581 85 Linköping, Sweden

Hazem Khalaf - Cardiovascular Research Centre, School of Medical Sciences, Örebro University, SE-703 62 Örebro, Sweden

Cristiane S. Farinas - Embrapa Instrumentation, 13561-206 São Carlos, SP, Brazil; Graduate Program of Chemical Engineering, Federal University of São Carlos, 13565-905 São Carlos, SP, Brazil; 10 orcid.org/0000-0002-9985-190X

Kristiina Oksman - Division of Materials Science, Department of Engineering Sciences and Mathematics, Luleå University of Technology, SE-971 87 Luleå, Sweden; Mechanical \& Industrial Engineering, University of Toronto, Toronto, Ontario M5S 3G8, Canada; (1) orcid.org/00000003-4762-2854

Complete contact information is available at:

https://pubs.acs.org/10.1021/acs.biomac.1c00215

\section{Author Contributions}

The manuscript was written through the contributions of all authors. Nanofibers and hydrogel preparation, SEM analysis, microscopy imaging, absorption and integrity measurements, and mechanical testing were carried out by P.S. L.B. performed the AFM analysis. K.H., J.R., and J.J. performed the cytocompatibility experiments. H.K. carried out the antimicrobial activity measurements. C.S.F., K.O., and L.B. supervised, and K.O. was responsible for funding acquisition. All authors have given approval to the final version of the manuscript

\section{Funding}

This work was financially supported by the Swedish Foundation for Strategic Research within the HEALiX project [RMX18-0039]; Bio4Energy, a strategic research environment, appointed by the Swedish government; and KAW [2018.0451] and WWSC. Kempe Foundations is acknowledged for the funding of AFM equipment. This study was also financed in part by the Coordenação de Aperfeiçoamento de Pessoal de Nivel Superior, Brasil (CAPES), Finance Code 001 for providing a scholarship to P.S. to conduct this research in Sweden.

\section{Notes}

The authors declare no competing financial interest.

\section{ACKNOWLEDGMENTS}

The authors thank Mitul Kumar and Simon Jonasson for their assistance in zeta potential measurements and nanofiber production, respectively. 


\section{ABBREVIATIONS USED}

TEMPO, (2,2,6,6-tetramethylpiperidine-1-oxyl radical)-mediated oxidation; BSA, bovine serum albumin; SEM, scanning electron microscopy; AFM, atomic force microscopy; PBS, phosphate-buffered saline; S. aureus, Staphylococcus aureus; E. coli, Escherichia coli; GEO, ginger essential oil

\section{REFERENCES}

(1) Dhivya, S.; Padma, V. V.; Santhini, E. Wound Dressings - a Review. BioMedicine 2015, 5, No. 22.

(2) Zarrintaj, P.; Moghaddam, A. S.; Manouchehri, S.; Atoufi, Z.; Amiri, A.; Amirkhani, M. A.; Nilforoushzadeh, M. A.; Saeb, M. R.; Hamblin, M. R.; Mozafari, M. Can Regenerative Medicine and Nanotechnology Combine to Heal Wounds? The Search for the Ideal Wound Dressing. Nanomedicine 2017, 12, 2403-2422.

(3) Schultz, G. S.; Sibbald, R. G.; Falanga, V.; Ayello, E. A.; Dowsett, C.; Harding, K.; Romanelli, M.; Stacey, M. C.; Teot, L.; Vanscheidt, W. Wound Bed Preparation: A Systematic Approach to Wound Management. Wound Repair Regener. 2003, 11, S1-S28.

(4) Alvarez, O. M.; Mertz, P. M.; Eaglstein, W. H. The Effect of Occlusive Dressings on Collagen Synthesis and Re-Epithelialization in Superficial Wounds. J. Surg. Res. 1983, 35, 142-148.

(5) Dowett, C.; Ayello, E. TIME Principles of Chronic Wound Bed Preparation and Treatment. Br. J. Nurs. 2004, 13, S16-S23.

(6) Singh, M. R.; Saraf, S.; Vyas, A.; Jain, V.; Singh, D. Innovative Approaches in Wound Healing: Trajectory and Advances. Artif. Cells, Nanomed., Biotechnol. 2013, 41, 202-212.

(7) Okan, D.; Woo, K.; Ayello, E. A.; Sibbald, G. The Role of Moisture Balance in Wound Healing. Adv. Skin Wound Care 2007, 20, $39-53$.

(8) Slaughter, B. V.; Khurshid, S. S.; Fisher, O. Z.; Khademhosseini, A.; Peppas, N. A. Hydrogels in Regenerative Medicine. Adv. Mater. 2009, 21, 3307-3329.

(9) Caló, E.; Khutoryanskiy, V. V. Biomedical Applications of Hydrogels: A Review of Patents and Commercial Products. Eur. Polym. J. 2015, 65, 252-267.

(10) Hoffman, A. S. Hydrogels for Biomedical Applications. Adv. Drug Delivery Rev. 2012, 64, 18-23.

(11) Buchholz, F. L.; Graham, A. T. Modern Superabsorbent Polymer Technology, 1st ed.; Wiley-VCH: New York, 1997.

(12) Opt Veld, R. C.; Walboomers, X. F.; Jansen, J. A.; Wagener, F. A. D. T. G. Design Considerations for Hydrogel Wound Dressings: Strategic and Molecular Advances. Tissue Eng., Part B 2020, 26, 230248.

(13) Xiang, J.; Shen, L.; Hong, Y. Status and Future Scope of Hydrogels in Wound Healing: Synthesis, Materials and Evaluation. Eur. Polym. J. 2020, 130, No. 109609.

(14) Isogai, A.; Bergström, L. Preparation of Cellulose Nanofibers Using Green and Sustainable Chemistry. Curr. Opin. Green Sustainable Chem. 2018, 12, 15-21.

(15) Wu, C. N.; Fuh, S. C.; Lin, S. P.; Lin, Y. Y.; Chen, H. Y.; Liu, J. M.; Cheng, K. C. TEMPO-Oxidized Bacterial Cellulose Pellicle with Silver Nanoparticles for Wound Dressing. Biomacromolecules 2018, 19, 544-554.

(16) Cheng, F.; Liu, C.; Wei, X.; Yan, T.; Li, H.; He, J.; Huang, Y. Preparation and Characterization of 2,2,6,6-Tetramethylpiperidine-1Oxyl (TEMPO)-Oxidized Cellulose Nanocrystal/Alginate Biodegradable Composite Dressing for Hemostasis Applications. ACS Sustainable Chem. Eng. 2017, 5, 3819-3828.

(17) Gao, H.; Zhong, Z.; Xia, H.; Hu, Q.; Ye, Q.; Wang, Y.; Chen, L.; Du, Y.; Shi, X.; Zhang, L. Construction of Cellulose Nanofibers/ Quaternized Chitin/Organic Rectorite Composites and Their Application as Wound Dressing Materials. Biomater. Sci. 2019, 7, $2571-2581$

(18) Nordli, H. R.; Pukstad, B.; Chinga-Carrasco, G.; Rokstad, A. M. Ultrapure Wood Nanocellulose - Assessments of Coagulation and Initial Inflammation Potential. ACS Appl. Bio Mater. 2019, 2, 11071118.
(19) Cheng, H.; Xiao, D.; Tang, Y.; Wang, B.; Feng, X.; Lu, M.; Vancso, G. J.; Sui, X. Sponges with Janus Character from Nanocellulose: Preparation and Applications in the Treatment of Hemorrhagic Wounds. Adv. Healthc. Mater. 2020, 9, 1-8.

(20) Curvello, R.; Raghuwanshi, V. S.; Garnier, G. Engineering Nanocellulose Hydrogels for Biomedical Applications. Adv. Colloid Interface Sci. 2019, 267, 47-61.

(21) Ferdous, Z.; Nemmar, A. Health Impact of Silver Nanoparticles: A Review of the Biodistribution and Toxicity Following Various Routes of Exposure. Int. J. Mol. Sci. 2020, 21, 2375.

(22) Li, J.; Cha, R.; Mou, K.; Zhao, X.; Long, K.; Luo, H.; Zhou, F.; Jiang, X. Nanocellulose-Based Antibacterial Materials. Adv. Healthc. Mater. 2018, 7, No. 1800334.

(23) Ali, B. H.; Blunden, G.; Tanira, M. O.; Nemmar, A. Some Phytochemical, Pharmacological and Toxicological Properties of Ginger (Zingiber officinale Roscoe): A Review of Recent Research. Food Chem. Toxicol. 2008, 46, 409-420.

(24) Nicoll, R.; Henein, M. Y. Ginger (Zingiber officinale Roscoe): A Hot Remedy for Cardiovascular Disease? Int. J. Cardiol. 2009, 131, 408-409.

(25) Sivasothy, Y.; Chong, W. K.; Hamid, A.; Eldeen, I. M.; Sulaiman, S. F.; Awang, K. Essential Oils of Zingiber officinale Var. Rubrum Theilade and Their Antibacterial Activities. Food Chem. 2011, 124, 514-517.

(26) Nile, S. H.; Park, S. W. Chromatographic Analysis, Antioxidant, Anti-Inflammatory, and Xanthine Oxidase Inhibitory Activities of Ginger Extracts and Its Reference Compounds. Ind. Crops Prod. 2015, $70,238-244$.

(27) Semwal, R. B.; Semwal, D. K.; Combrinck, S.; Viljoen, A. M. Gingerols and Shogaols: Important Nutraceutical Principles from Ginger. Phytochemistry 2015, 117, 554-568.

(28) Butt, M. S.; Sultan, M. T. Ginger and Its Health Claims: Molecular Aspects. Crit. Rev. Food Sci. Nutr. 2011, 51, 383-393.

(29) Singh, G.; Kapoor, I. P. S.; Singh, P.; de Heluani, C. S.; de Lampasona, M. P.; Catalan, C. A. N. Chemistry, Antioxidant and Antimicrobial Investigations on Essential Oil and Oleoresins of Zingiber officinale. Food Chem. Toxicol. 2008, 46, 3295-3302.

(30) El-Baroty, G. S.; Abd El-Baky, H. H.; Farag, R. S.; Saleh, M. A. Characterization of Antioxidant and Antimicrobial Compounds of Cinnamon and Ginger Essential Oils. Afr. J. Biochem. Res. 2010, 4, $167-174$.

(31) Sang, S.; Snook, H. D.; Tareq, F. S.; Fasina, Y. Precision Research on Ginger: The Type of Ginger Matters. J. Agric. Food Chem. 2020, 68, 8517-8523.

(32) Jacob, J.; Peter, G.; Thomas, S.; Haponiuk, J. T.; Gopi, S. Chitosan and Polyvinyl Alcohol Nanocomposites with Cellulose Nanofibers from Ginger Rhizomes and Its Antimicrobial Activities. Int. J. Biol. Macromol. 2019, 129, 370-376.

(33) Jacob, J.; Haponiuk, J. T.; Thomas, S.; Peter, G.; Gopi, S. Use of Ginger Nanofibers for the Preparation of Cellulose Nanocomposites and Their Antimicrobial Activities. Fibers 2018, 6, No. 79.

(34) Jacob, J.; Peter, G.; Thomas, S.; Haponiuk, J. T.; Gopi, S. InSitu Synthesis and Characterization of Biocompatible Magnetic Ginger Nanofiber Composites for Copper (II) Removal from Water. Mater. Today Commun. 2019, 21, No. 100690.

(35) Abral, H.; Ariksa, J.; Mahardika, M.; Handayani, D.; Aminah, I.; Sandrawati, N.; Pratama, A. B.; Fajri, N.; Sapuan, S. M.; Ilyas, R. A. Transparent and Antimicrobial Cellulose Film from Ginger Nanofiber. Food Hydrocoll. 2020, 98, No. 105266.

(36) Abral, H.; Ariksa, J.; Mahardika, M.; Handayani, D.; Aminah, I.; Sandrawati, N.; Sugiarti, E.; Muslimin, A. N.; Rosanti, S. D. Effect of Heat Treatment on Thermal Resistance, Transparency and Antimicrobial Activity of Sonicated Ginger Cellulose Film. Carbohydr. Polym. 2020, 240, No. 116287.

(37) Abral, H.; Ariksa, J.; Mahardika, M.; Handayani, D.; Aminah, I.; Sandrawati, N.; Sapuan, S. M.; Ilyas, R. A. Highly Transparent and Antimicrobial PVA Based Bionanocomposites Reinforced by Ginger Nanofiber. Polym. Test. 2020, 81, No. 106186. 
(38) Wang, D.; Yu, H.; Fan, X.; Gu, J.; Ye, S.; Yao, J.; Ni, Q. High Aspect Ratio Carboxylated Cellulose Nanofibers Cross-Linked to Robust Aerogels for Superabsorption-Flocculants: Paving Way from Nanoscale to Macroscale. ACS Appl. Mater. Interfaces 2018, 10, 20755-20766.

(39) Wang, D.-C.; Yu, H.-Y.; Qi, D.; Ramasamy, M.; Yao, J.; Tang, F.; Tam, K. M. C.; Ni, Q. Supramolecular Self-Assembly of 3D Conductive Cellulose Nanofiber Aerogels for Flexible Supercapacitors and Ultrasensitive Sensors. ACS Appl. Mater. Interfaces 2019, 11, 24435-24446.

(40) TAPPI, T. 204 Cm-97. Solvent Extr. wood pulp, 1997. https:// www.tappi.org/content/sarg/t204.pdf (accessed Oct 15, 2020).

(41) Wise, L. E. Chlorite Holocellulose, Its Fractionation and Bearing on Summative Wood Analysis and on Studies on the Hemicelluloses. Pap. Trade 1946, 122, 35-43.

(42) TAPPI, N. T203 Cm-99.“. Alpha-, beta-and gamma-cellulose pulp, 1999. https://imisrise.tappi.org/TAPPI/Products/01/T/0104T203. aspx (accessed Oct 15, 2020).

(43) Tappi, T. 222 Om-02: Acid-Insoluble Lignin in Wood and Pulp. 2002-2003 TAPPI Test Methods, 2002. https://www.tappi.org/ content/SARG/T222.pdf (accessed Oct 15, 2020).

(44) Saito, T.; Hirota, M.; Tamura, N.; Kimura, S.; Fukuzumi, H.; Heux, L.; Isogai, A. Individualization of Nano-Sized Plant Cellulose Fibrils by Direct Surface Carboxylation Using TEMPO Catalyst under Neutral Conditions. Biomacromolecules 2009, 10, 1992-1996.

(45) Rheinwatd, J. G.; Green, H. Seria Cultivation of Strains of Human Epidemal Keratinocytes: The Formation Keratinizin Colonies from Single Cell Is. Cell 1975, 6, 331-343.

(46) Zaki, F. A.; Abdullah, I.; Ahmad, I. The Physical and Chemical Natures of Cellulose Extracted from Torch Ginger Stems. Int. J. Mater. Eng. Innov. 2014, 5, 48-60.

(47) Schwertner, H. A.; Rios, D. C.; Pascoe, J. E. Variation in Concentration and Labeling of Ginger Root Dietary Supplements. Obstet. Gynecol. 2006, 107, 1337-1343.

(48) Reyes, F. G. R.; D’Appolonia, B. L.; Ciacco, C. F.; Montgomery, M. W. Characterization of Starch from Ginger Root (Zingiber officinale). Starch 1982, 34, 40-44.

(49) Braga, M. E. M.; Moreschi, S. R. M.; Meireles, M. A. A. Effects of Supercritical Fluid Extraction on Curcuma Longa L. and Zingiber officinale R. Starches. Carbohydr. Polym. 2006, 63, 340-346.

(50) Bartos, A.; Anggono, J.; Farkas, ÁE.; Kun, D.; Soetaredjo, F. E.; Móczó, J.; Antoni; Purwaningsih, H.; Pukánszky, B. Alkali Treatment of Lignocellulosic Fibers Extracted from Sugarcane Bagasse: Composition, Structure, Properties. Polym. Test. 2020, 88, No. 106549.

(51) Policegoudra, R. S.; Aradhya, S. M. Structure and Biochemical Properties of Starch from an Unconventional Source-Mango Ginger (Curcuma Amada Roxb.) Rhizome. Food Hydrocolloids 2008, 22, $513-519$.

(52) Jonasson, S.; Bünder, A.; Niittylä, T.; Oksman, K. Isolation and Characterization of Cellulose Nanofibers from Aspen Wood Using Derivatizing and Non-Derivatizing Pretreatments. Cellulose 2020, 27, 185-203.

(53) Holback, H.; Yeo, Y.; Park, K. Hydrogel Swelling Behavior and Its Biomedical Applications; Woodhead Publishing Limited, 2011.

(54) Sood, A.; Granick, M. S.; Tomaselli, N. L. Wound Dressings and Comparative Effectiveness Data. Adv. Wound Care 2014, 3, 511529.

(55) Pourjavadi, A.; Ayyari, M.; Amini-Fazl, M. S. Taguchi Optimized Synthesis of Collagen-g-Poly(Acrylic Acid)/Kaolin Composite Superabsorbent Hydrogel. Eur. Polym. J. 2008, 44, 1209-1216.

(56) Horie, K.; Barón, M.; Fox, R. B.; He, J.; Hess, M.; Kahovec, J.; Kitayama, T.; Kubisa, P.; Maréchal, E.; Mormann, W.; et al. Definitions of Terms Relating to Reactions of Polymers and to Functional Polymeric Materials (IUPAC Recommendations 2003). Pure Appl. Chem. 2004, 76, 889-906.
(57) Alam, M. N.; Islam, M. S.; Christopher, L. P. Sustainable Production of Cellulose-Based Hydrogels with Superb Absorbing Potential in Physiological Saline. ACS Omega 2019, 4, 9419-9426.

(58) Zhang, J.; Xiao, H.; Li, N.; Ping, Q.; Zhang, Y. Synthesis and Characterization of Super-Absorbent Hydrogels Based on Hemicellulose. J. Appl. Polym. Sci. 2015, 132, 2-7.

(59) Yang, J.; Fang, L.; Tan, T. Synthesis and Characterization of Superabsorbent Hydrogels Composites Based on Polysuccinimide. J. Appl. Polym. Sci. 2006, 102, 550-557.

(60) Peppas, N. A.; Bures, P.; Leobandung, W.; Ichikawa, H. Hydrogels in Pharmaceutical Formulations. Eur. J. Pharm. Biopharm. 2000, 50, 27-46.

(61) Yon, R. J. Chromatography of Lipophilic Proteins on Adsorbents Containing Mixed Hydrophobic and Ionic Groups. Biochem. J. 1972, 126, 765-767.

(62) Böhme, U.; Scheler, U. Effective Charge of Bovine Serum Albumin Determined by Electrophoresis NMR. Chem. Phys. Lett. 2007, 435, 342-345.

(63) Kono, H.; Oeda, I.; Nakamura, T. The Preparation, Swelling Characteristics, and Albumin Adsorption and Release Behaviors of a Novel Chitosan-Based Polyampholyte Hydrogel. React. Funct. Polym. 2013, 73, 97-107.

(64) Oksman, K.; Aitomäki, Y.; Mathew, A. P.; Siqueira, G.; Zhou, Q.; Butylina, S.; Tanpichai, S.; Zhou, X.; Hooshmand, S. Review of the Recent Developments in Cellulose Nanocomposite Processing. Composites, Part A 2016, 83, 2-18.

(65) Berglund, L.; Anugwom, I.; Hedenström, M.; Aitomäki, Y.; Mikkola, J.-P.; Oksman, K. Switchable Ionic Liquids Enable Efficient Nanofibrillation of Wood Pulp. Cellulose 2017, 24, 3265-3279.

(66) Jonoobi, M.; Mathew, A. P.; Oksman, K. Producing Low-Cost Cellulose Nanofiber from Sludge as New Source of Raw Materials. Ind. Crops Prod. 2012, 40, 232-238.

(67) Benselfelt, T.; Engström, J.; Wågberg, L. Supramolecular Double Networks of Cellulose Nanofibrils and Algal Polysaccharides with Excellent Wet Mechanical Properties. Green Chem. 2018, 20, 2558-2570.

(68) Benselfelt, T.; Wågberg, L. Unidirectional Swelling of Dynamic Cellulose Nanofibril Networks: A Platform for Tunable Hydrogels and Aerogels with 3D Shapeability. Biomacromolecules 2019, 20, 2406-2412.

(69) Zhao, M.; Ansari, F.; Takeuchi, M.; Shimizu, M.; Saito, T.; Berglund, L. A.; Isogai, A. Nematic Structuring of Transparent and Multifunctional Nanocellulose Papers. Nanoscale Horiz. 2018, 3, 2834.

(70) Iwamoto, S.; Abe, K.; Yano, H. The Effect of Hemicelluloses on Wood Pulp Nanofibrillation and Nanofiber Network Characteristics. Biomacromolecules 2008, 9, 1022-1026.

(71) Hakkarainen, T.; Koivuniemi, R.; Kosonen, M.; EscobedoLucea, C.; Sanz-Garcia, A.; Vuola, J.; Valtonen, J.; Tammela, P.; Mäkitie, A.; Luukko, K.; Yliperttula, M.; Kavola, H. Nanofibrillar Cellulose Wound Dressing in Skin Graft Donor Site Treatment. J. Control. Release 2016, 244, 292-301.

(72) Sun, F.; Nordli, H. R.; Pukstad, B.; Kristofer Gamstedt, E.; Chinga-Carrasco, G. Mechanical Characteristics of NanocellulosePEG Bionanocomposite Wound Dressings in Wet Conditions. J. Mech. Behav. Biomed. Mater. 2017, 69, 377-384.

(73) Wang, Q.; Yao, Q.; Liu, J.; Sun, J.; Zhu, Q.; Chen, H. Processing Nanocellulose to Bulk Materials: A Review; Springer: Netherlands, 2019; Vol. 26.

(74) Sehaqui, H.; Liu, A.; Zhou, Q.; Berglund, L. A. Fast Preparation Procedure for Large, Flat Cellulose and Cellulose/Inorganic Nanopaper Structures. Biomacromolecules 2010, 11, 2195-2198.

(75) Qing, Y.; Sabo, R.; Wu, Y.; Zhu, J. Y.; Cai, Z. Self-Assembled Optically Transparent Cellulose Nanofibril Films: Effect of Nanofibril Morphology and Drying Procedure. Cellulose 2015, 22, 1091-1102.

(76) Du, H.; Liu, W.; Zhang, M.; Si, C.; Zhang, X.; Li, B. Cellulose Nanocrystals and Cellulose Nanofibrils Based Hydrogels for Biomedical Applications. Carbohydr. Polym. 2019, 209, 130-144. 
(77) Pailler-Mattei, C.; Bec, S.; Zahouani, H. In Vivo Measurements of the Elastic Mechanical Properties of Human Skin by Indentation Tests. Med. Eng. Phys. 2008, 30, 599-606.

(78) Ghasemzadeh, A.; Jaafar, H. Z. E.; Rahmat, A. Changes in Antioxidant and Antibacterial Activities as Well as Phytochemical Constituents Associated with Ginger Storage and Polyphenol Oxidase Activity. BMC Complementary Altern. Med. 2016, 16, No. 382.

(79) Weishaupt, R.; Zünd, J. N.; Heuberger, L.; Zuber, F.; Faccio, G.; Robotti, F.; Ferrari, A.; Fortunato, G.; Ren, Q.; Maniura-Weber, K.; Guex, A. G. Antibacterial, Cytocompatible, Sustainably Sourced: Cellulose Membranes with Bifunctional Peptides for Advanced Wound Dressings. Adv. Healthcare Mater. 2020, 9, No. 1901850.

(80) Mi, G.; Shi, D.; Wang, M.; Webster, T. J. Reducing Bacterial Infections and Biofilm Formation Using Nanoparticles and Nanostructured Antibacterial Surfaces. Adv. Healthcare Mater. 2018, 7, No. 1800103.

(81) Chen, Y.; Xu, M.; Zhang, J.; Ma, J.; Gao, M.; Zhang, Z.; Xu, Y.; Liu, S. Genome-Wide DNA Methylation Variations upon Exposure to Engineered Nanomaterials and Their Implications in Nanosafety Assessment. Adv. Mater. 2017, 29, No. 1604580.

(82) Pusnik, M.; Imeri, M.; Deppierraz, G.; Bruinink, A.; Zinn, M. The Agar Diffusion Scratch Assay - A Novel Method to Assess the Bioactive and Cytotoxic Potential of New Materials and Compounds. Sci. Rep. 2016, 6, No. 20854. 